\title{
Real-time feasibility of nonlinear model predictive control for semi- batch reactors subject to uncertainty and disturbances
}

\author{
Harvey Arellano-Garcia ${ }^{\mathrm{a}, \mathrm{b}}$, Tilman Barz ${ }^{\mathrm{c}}$, Bogdan Dorneanu ${ }^{\mathrm{a}}$, Vassilios S. Vassiliadis ${ }^{\mathrm{d},{ }^{*}}$ \\ a Department of Chemical and Process Engineering, University of Surrey, Guildford, GU2 7XH, United Kingdom \\ b LS Prozess- und Anlagentechnik, Brandenburgische Technische Universität Cottbus-Senftenberg, Cottbus, D- \\ 03046, Germany \\ ${ }^{c}$ AlT Austrian Institute of Technology GmbH, Center for Energy, Vienna, 1210, Austria

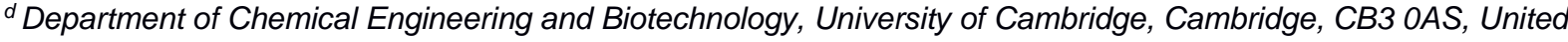 \\ Kingdom
}

*Corresponding author

\section{Abstract}

This paper presents two nonlinear model predictive control based methods for solving closedloop stochastic dynamic optimisation problems, ensuring both robustness and feasibility with respect to state output constraints. The first one is a new deterministic approach, using the waitand-see strategy. The key idea is to specifically anticipate violation of output hard-constraints, which are strongly affected by instantaneous disturbances, by backing off of their bounds along the moving horizon. The second method is a stochastic approach to solve nonlinear chanceconstrained dynamic optimisation problems under uncertainties. The key aspect is the explicit consideration of the stochastic properties of both exogenous and endogenous uncertainties in the problem formulation (here-and-now strategy). The approach considers a nonlinear relation between uncertain inputs and the constrained state outputs. The performance of the proposed methodologies is assessed via an application to a semi-batch reactor under safety constraints, involving strongly exothermic reactions.

Keywords: NMPC, Output-Constraints, Chance-Constraints, Dynamic Real Time Optimisation, Batch Processes, Safety.

\section{Introduction}

Model-based process control has become significant during the last few decades (Morari \& Lee, 1999; Qin \& Badgwell, 2003; Lee, 2011; Darby \& Nikolaou, 2012; Ellis et al., 2014; Mayne, 2014; Forbes et al., 2015; Saltik et al., 2018). However, for a quantitative understanding and control of time-varying phenomena in process systems, it is essential to relate the observed dynamical behaviour to mathematical models. Due to the generally limited quality and quantity of inputoutput data used to fit the model, the model will not be an exact representation of the true process. Thus, the practical implementation of model-based techniques often leads to a significant discrepancy between reality and simulation. These models usually depend on a number of parameters whose values are either entirely unknown or only known to a limited degree of reliability. Furthermore, often only a part of the system's dynamics can be measured. Therefore, 
a plant model unavoidably involves uncertainties. They are either endemic due to the external disturbances or introduced into the model to account for imprecisely known dynamics.

These uncertainties or disturbances are often multivariate and correlated stochastic sequences. Moreover, the use of feedback control in order to compensate uncertainties cannot ensure satisfaction of constraints on open-loop variables. Thus, a closed-loop control requires on-line measured values of controlled variables. Many variables in engineering practice cannot, though, be measured on-line. These variables often represent the product quality and, thus, their control is highly desired and of great importance. To overcome this limitation, measurable variables are chosen as controlled variables in order to control the product quality indirectly. This concept is schematically illustrated in Figure 1. Here, the measurable output variables $y$ will be controlled at their set-points $y^{S P}$ by using the control variables $u$. On the other hand, control of $y^{C}$ is preferred, but, due to the lack of on-line measurement it has to be open-loop. In these cases, $y^{C}$ needs to be constrained instead of $y$.

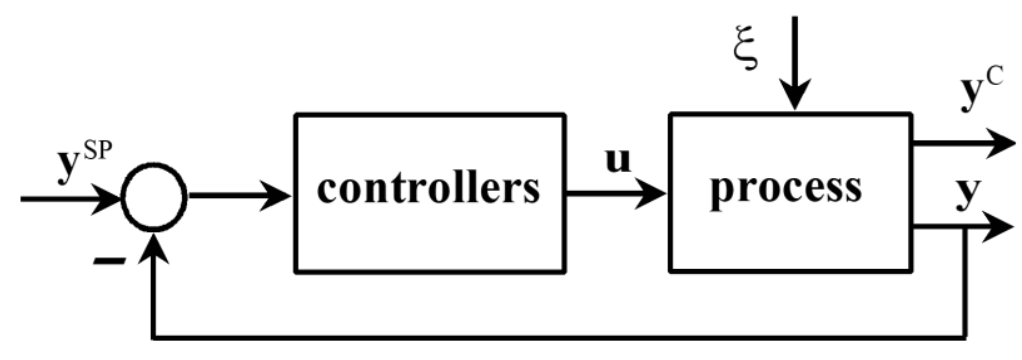

Figure 1: The open-closed control framework

To guarantee the product quality, the common procedure in industrial practice is to select an extremely conservative set-point value. This implies that the product quality will be unnecessarily much higher than specified and, thus, the operation costs will also be much higher than necessary as a result. Consequently, an optimal group of set-points for the controllers is needed which are neither too conservative, nor too aggressive. In addition, constrained variables for safety or environmental considerations are often monitored but not closed-loop controlled. Thus, it is required to evaluate the probability of violating these constraints at the decided operating point.

The restricted industrial acceptance of model-based optimisation techniques is caused by the availability of detailed dynamic models (Forbes et al., 2015). Their lack of reliability together with the presence of uncertainty has motivated the investigation of process improvement. For this purpose, in this paper, chance constrained optimisation is proposed, i.e. the objective function (e.g. costs) is improved and the constraints with regard to $y^{c}$ are then to be satisfied with a predefined confidence level. Thus, unlike the problem definition above, where controls are decision variables, in the closed-loop framework the set-points of the measurable outputs are defined as decision variables. The controls will react based on the realisation of the uncertain inputs and, thus, they are uncertain variables. Consequently, the consideration of uncertainties/disturbances and their stochastic properties in optimisation approaches are necessary for robust process control. 


\section{Problem statement}

Batch processing provides greater flexibility in the production of speciality and pharmaceutical chemicals. The trend in the chemical industry towards high added value products has increased interest in the optimal, model-based control of batch processes. In contrast to continuous processes which have been subject to several rigorous optimisation studies, batch and semibatch reactors are often still operated using recipes which are based on heuristics and experience (Pahija et al., 2013). Due to its ability to include constraints directly in the computation of the control moves, nonlinear model predictive control (NMPC) offers advantages for the optimal operation of transient chemical plants (Morari \& Lee, 1999). However, numerous robust predictive controllers suffer from excessively conservative control because they rely upon open-loop predictions of future system uncertainty (Kothare et al., 1996; Bemporad \& Morari, 1999; Alessio \& Bemporad, 2009; Kähm \& Vassiliadis, 2018a, b, c).

Open-loop predictions overestimate the uncertainty in future outputs (Lee \& Yu, 1997; Ma et al., 1999; Nagy and Braatz, 2004). Furthermore, since the true process optimum frequently lies on a boundary of the feasible region defined by one or more active constraints, the process is inevitably forced into an infeasible region due to the uncertainty in the parameters, external disturbances, and measurement errors (Chisci et al., 2001; Yu et al., 2014). Thus, the risk of infeasibility at every sampling instant represents another critical issue in model predictive control (MPC) schemes. Hence, the formulation of soft constraints has become common to handle state output constraints, in which penalty terms concerning the constraints are included in the objective function. This prevents infeasibility problems by allowing violations of the constraints (Mayne, 2000; Mayne et al., 2000; Mayne, 2016).

On the other hand, approaches based upon relaxation are, in fact, inapplicable for processes with strict safety restrictions which are not supposed to be violated at any time point. Deterministic approaches for handling robustness in MPC (Mhaskar et al., 2005; Saltik et al., 2018) and to ensuring state constraint satisfaction via modification of the constraints for steady state processes (Dubljevic et al., 2005) have been proposed. Besides, although NMPC can inherently exhibit a certain degree of robustness (De Nicolao et al., 2000; Zavala \& Biegler, 2009; Griffith et al., 2018), for safety-critical transient processes, however, an explicit consideration of uncertainty and disturbances is needed.

\subsection{Motivating case study}

A strongly exothermic series reaction conducted in a non-isothermal batch reactor (Figure 2) is considered. The reaction mechanism considers a second-order kinetics for the first reaction producing $B$ from $A$, and a first-order kinetics for the undesirable consecutive reaction converting $B$ to $C$. The reaction scheme is given as follows:

$$
2 \mathrm{~A} \stackrel{\mathrm{K} 1}{\longrightarrow} \mathrm{B} \stackrel{\mathrm{K} 2}{\longrightarrow} \mathrm{C}
$$


The intermediate product $B$ is deemed to be the desired product. In order to prevent the risk of runways, batch reactors are usually equipped with two cooling systems, a jacket around the reactor tank and a coil inside the reactor. In this case study, only the dynamics of the jacket cooling system is explicitly considered while the temperature of the cooling system inside is used as an operational degree of freedom. The rector flowsheet is shown in Figure 2.

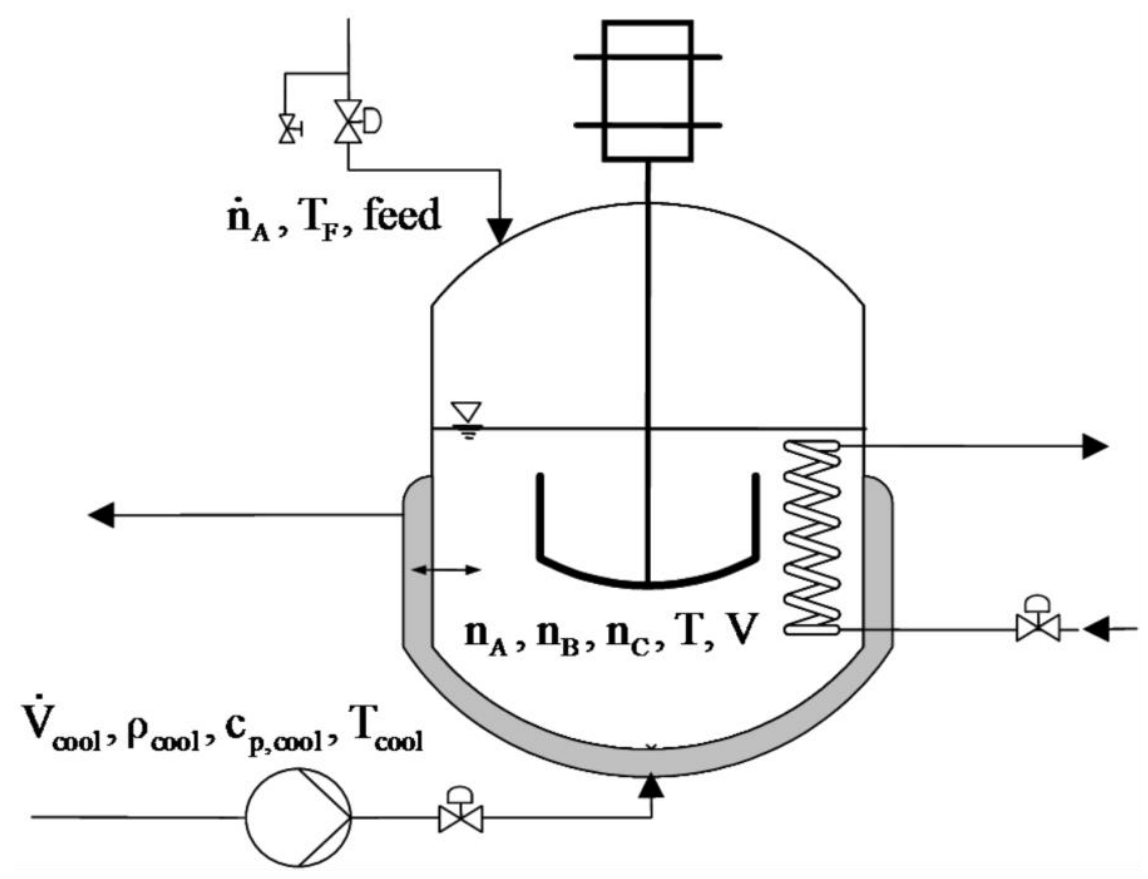

Figure 2: Scheme of the semi-batch reactor and its cooling system

In addition, for safety reasons, the potentially hazardous process is operated in a fed-batch manner. However, in industrial practice, the simple feeding strategy with a constant dosing rate over the entire batch time is commonly used.

\section{A NMPC-based on-line optimisation approach}

For this purpose, a NMPC scheme is proposed to solve closed-loop dynamic optimisation problems, ensuring both robustness and feasibility with respect to output constraints. The main concept lies in the consideration of unknown and unexpected disturbances in advance. The novel deterministic approach is based on the wait-and-see strategy. The key idea is here to anticipate violation of output hard-constraints (safety restrictions), which are strongly affected by instantaneous disturbances. Thus, in order to guarantee that the determined optimal operation remains safe, safety restrictions are needed to be incorporated explicitly in the NMPC optimisation problem model.

Even though operation at this optimum is preferred, it usually cannot be accomplished with simultaneous fulfilment of all constraints, due to the effect of external disturbances. Thus, in this section, an NMPC based approach is proposed to implement such an optimal strategy remaining safe despite disturbances. 
The consideration of these output constraints for the control problem in the limited horizon does not naturally imply that a feasible operation can be guaranteed at each time point. Poorly defined constraints within the moving horizon can lead to a deadlock situation. That means that the system is manoeuvred into a situation where the problem is infeasible and cannot be solved with the given optimisation variables and their bounds (Helbig et al., 1998). In connection with batch reactors a deadlock situation arises when a high educt accumulation takes place in the reactor, leading to a heat development which exceeds the system cooling capacity. By this effect, the reactor can no longer be controlled, and a runaway reaction results.

In order to prevent such situations, a predictive optimisation is necessary. This is however limited through the horizon length and, thus, is not necessarily enough in order to hold the process within a feasible operating region. Previous studies on this issue show that certain restrictions can be formulated so as to prevent a deadlock situation. One possibility will be to work with special path constraints which consider worst case scenarios. Another alternative assuring feasibility can also be the restriction of the allowable deviation from the set-point trajectory. In this work, the main aim is to meet the safety constraints under all circumstances. Therefore, deviations from the originally determined trajectories to the possible disadvantage of the economic objectives are accepted.

\subsection{Dynamic adaptive back-off strategy}

Since the true process optimum often lies on a boundary of the feasible region defined by one or more active constraints, the process is forced into an infeasible region due to the uncertainty in the parameters, external disturbances, and measurement errors. Thus, the risk of infeasibility at every sampling instant represents another critical issue in MPC. Hence, the formulation of soft constraints has become common to handle state and output constraints, in which penalty terms on the constraints are included in the objective function. This prevents infeasibility problems by allowing violations of the constraints. On the other hand, approaches based upon relaxation are, in fact, inapplicable for processes with safety restrictions which are not supposed to be violated at any time point (thus comprising hard constraints, as opposed to previous ones which are soft constraints). Besides, although NMPC can inherently exhibit a certain degree of robustness, for safety-critical transient processes an explicit consideration of uncertainty and disturbances is strictly required.

This issue can be explained with an assumed trajectory of the reactor temperature ( $T$-model) in Figure 3. This temperature is calculated with the controller model and it depends on the initial value $T\left(t_{0}\right)$ and on the cooling flow rate strategy within the horizon $\left(0 \leq t \leq T_{P}\right)$. The cooling flow rate is determined through solution of the optimisation problem such that the discrete values of the reactor temperature are feasible at the end of each interval, i.e. the maximum allowable reactor temperature $T_{\max }$ is not exceeded. In Figure 3, the values $T(j=1)$ and $T(j=2)$ lie on the 
boundary of the feasible region, which means that the constraint is active. The implementation is represented in Figure 3 at the bottom on the left.
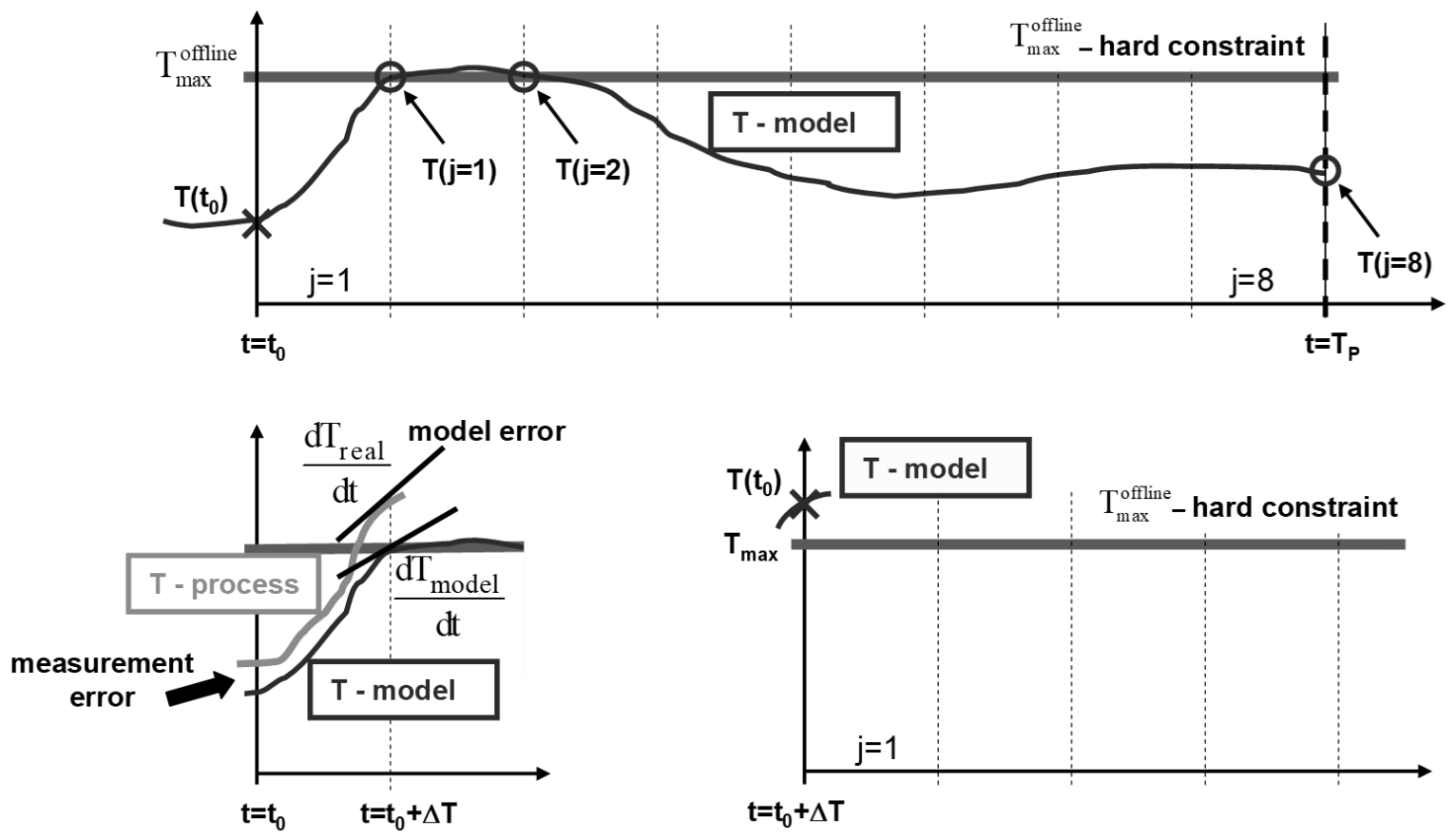

Figure 3: Course of the reactor temperature within the horizon

The reactor temperature at the end of the first interval differs from the predicted temperature due to the measurement and model errors, and the hard-constraint is violated. For the sake of demonstration, a poor direction of action has been chosen. This does not mean that a constraint violation is always unavoidable.

The third diagram on the right in Figure 3 represents the issue which corresponds to the initial value for the optimisation problem in the next interval. This value is determined through measurement and does not necessarily correspond to the actual process state. In this case, a feasible problem solution means that the decision variable (cooling flow rate) is selected in such a way that the reactor temperature will lie inside of the feasible region at the end of each interval. But, it is also possible that even the maximum system cooling capacity is not able to realise this demand. This results in the optimisation problem to become unsolvable. Thus, practical implementation of NMPC becomes difficult for any reasonably non-trivial nonlinear system (Mayne et al., 2000). However, as illustrated in Figure 3, critical issues are robustness and the feasibility of the optimisation problem, i.e. the presence of an input profile that satisfies the constraints.

Thus it may be necessary to back off from the nominal optimal value of the constraints which are difficult to measure or to control due to the poor dynamics. These so-called back-off strategies (Loeblein \& Perkins, 1999; Logist et al, 2011), in which the original path constraint is shifted to another value, so that the process always has a certain distrance to the original constraint (Puschke, et al. 2017). Various studies investigated their application (Visser et al, 2000; Soliman, 
et al., 2008; Galvanin, et al., 2009; Shi, et al., 2016; Aydin, et al., 2018; Koller, et al., 2018; Maussner \& Freund, 2018; Emenike, et al., 2019) by developing ways to tighten violated constraints and shrinks the feasible region such as the worst-case scenario of a given process will still be feasible despite variations in the constraints.

In order to guarantee robustness and feasibility with respect to output constraints, despite of uncertainties and unexpected disturbances, an adaptive dynamic back-off strategy is introduced into the optimisation problem to guarantee that the restrictions are not violated an ane point, in particular, in case of sudden cooling failure. These back-offs describe the distance between values for nominal optimal computed outputs and the corrections which add conservative distances to constraints (Barz et al, 2011). For this purpose, it is necessary to consider the impact of the uncertainties between the time points for re-optimisation and the resulting control re-setting by setting, in advance, the constraint bounds to be much more severe than the physical ones within the moving horizon.

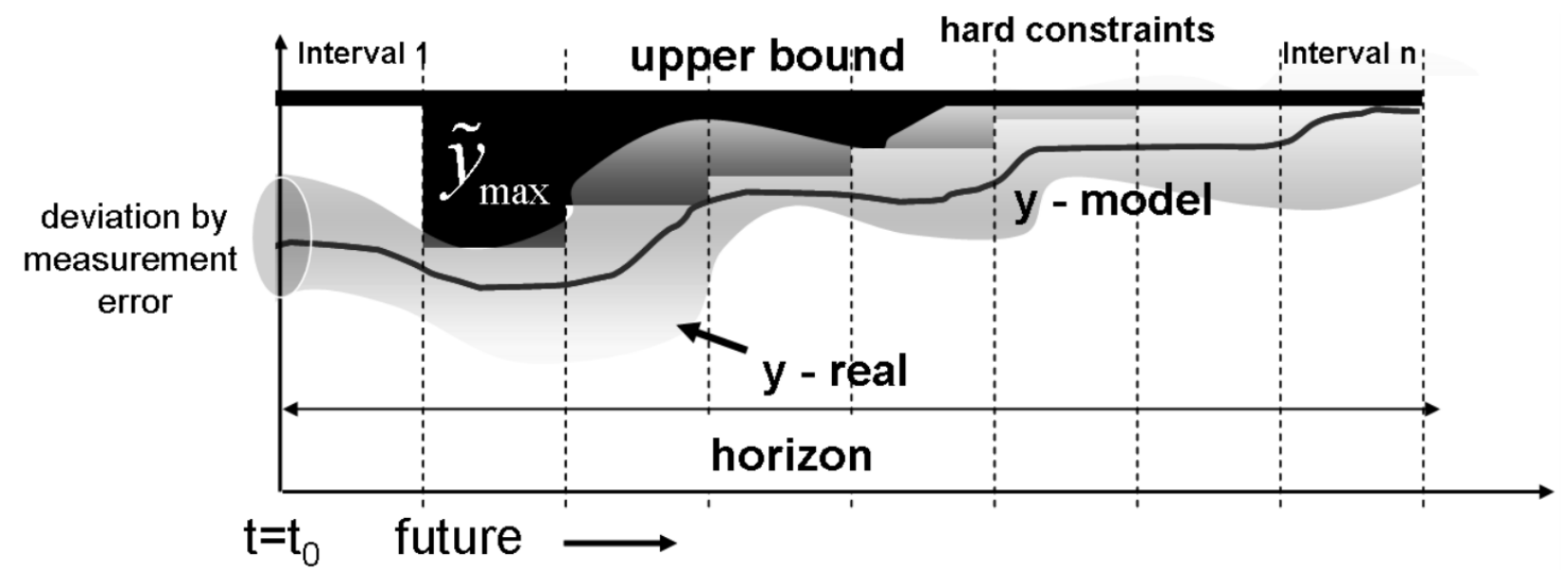

Figure 4: Back-off strategy within the moving horizon

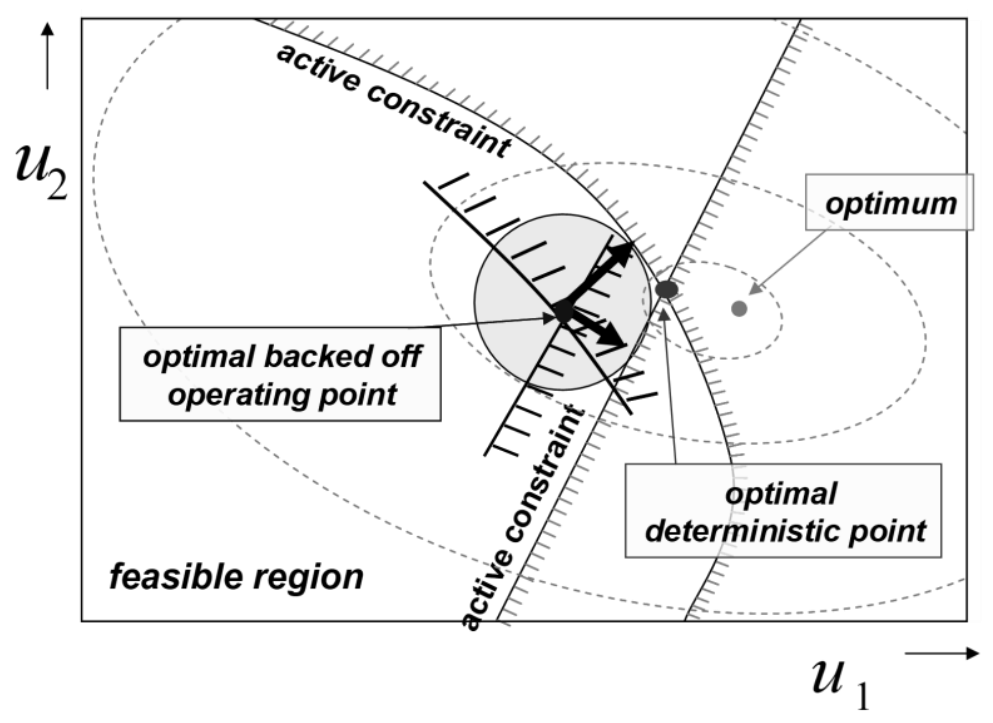

Figure 5: Back-off from active constraints 
Thus, as shown in Figures 4 and 5, the key idea of the approach is based on backing-off of these bounds with a decreasing degree of severity leading then to the generation of a trajectory which consists of the modified constraint bounds along the moving horizon (8 intervals). For the near future time points within the horizon, these limits (bounds) are more severe than the real physical constraints and will gradually be eased (e.g. logarithmically) for further time points. The trajectory of these bounds is dependent on the amount of measurement error and parameter variation including uncertainty.

In some cases, the true process optimum lies on the boundary of the feasible region defined by the active constraints. Due to the uncertainty in the parameters and the measurement errors, the process optimum and the set-point trajectory would be infeasible. By introducing a back-off from the active constraints in the optimisation, the region of the set-point trajectory is moved inside the feasible region of the process to ensure, on the one hand, feasible operation, and to operate the process, on the other hand, still as closely to the true optimum as possible (Figure 5). Consequently, the darkened area in Figure 4 illustrates the corrected bounds $\tilde{y}_{\max }$ of the hard constraints. Here, it should however be noted that due to the tighter bound at the computation of the previous horizon, the initial value at $t_{0}$ is rather far away from the constraint limit in the feasible area. Thus, in the first interval of the current moving horizon, the bound is set at the original physical limit to avoid infeasibility. The back-off adjustment starts from the second interval, i.e. from the time point on which the next re-optimisation begins. Since there will be more time points for re-optimisation and thus for compensating disturbances, for the further remaining intervals within the moving horizon $\tilde{y}_{\max }$ approaches to the original bound. The size of $\tilde{y}_{\max }$ strongly depends on parametric uncertainty, disturbances, and the deviation caused by measurement errors.

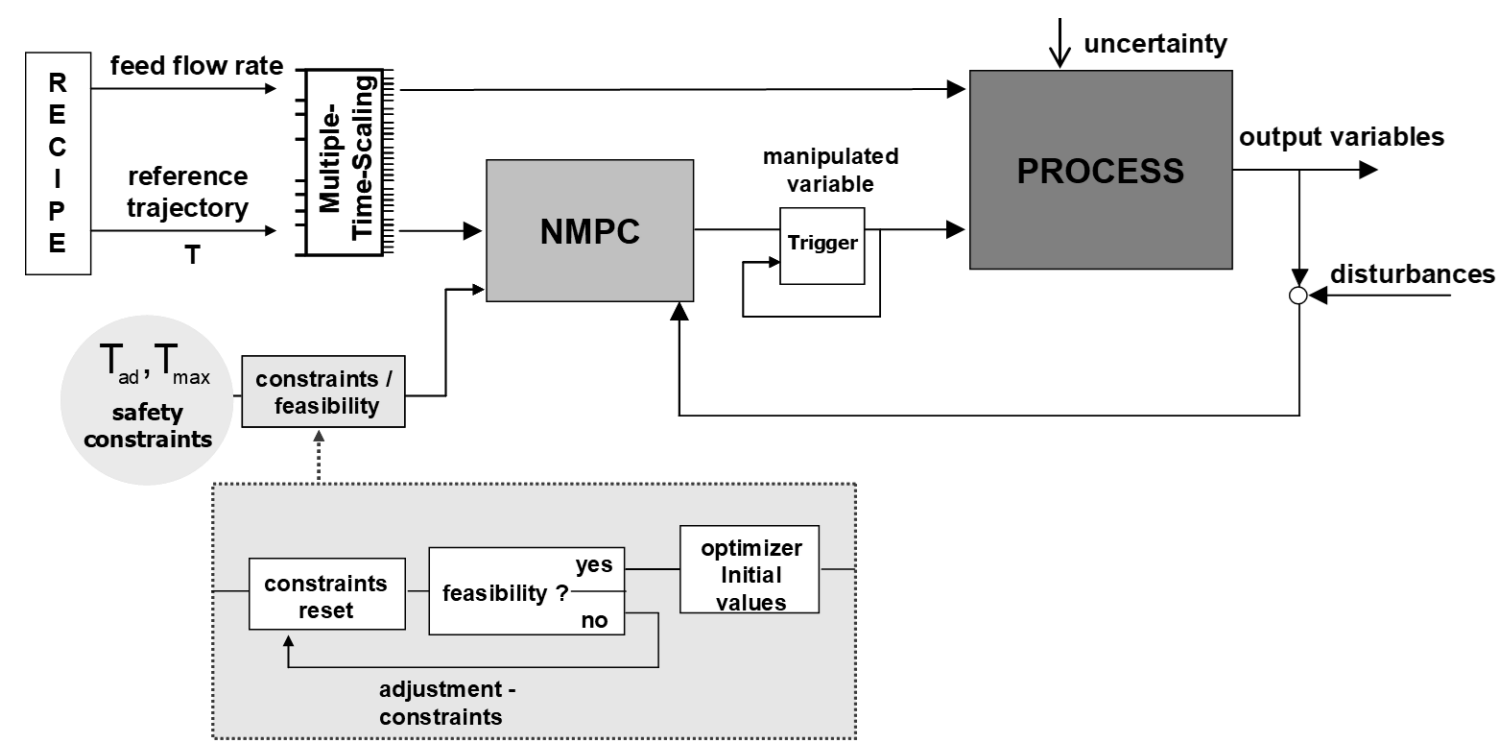

Figure 6: Closed-loop optimisation framework including hard output constraints

The developed closed-loop optimisation framework is depicted in Figure 6. The concept incorporates also a feasibility analysis with regard to the handling of safety constraints. For this 
purpose, the safety constraints are set at the beginning of each time step. By this means, the value of $\tilde{y}_{\max }$ can be verified through simulation.

In case the optimisation algorithm does not find a solution, a trigger is activated that holds the current control signal fixed also for the next interval. Another important issue is represented by the initial values for the optimisation. Here, the values of the open-loop optimisation are used as reference.

Furthermore, by means of simulation, the constraint tightening (back-off) within the horizon can be estimated. This depends, however, on the size and effect of the arising uncertainties. The back-off is also to be selected as small as possible in order not to lose optimisation potential. In case the required process knowledge is not available, then the back-off is determined in a conservative manner.

\subsection{A two-level strategy for optimisation based control}

The size of the dynamic operating region around the optimum (see Figure 6) is affected by fast disturbances. These are however, efficiently rejected by the proposed regulatory NMPC-based approach. On the other hand, there are, in fact, slowly time-varying non-zero mean disturbances or drifting model parameters which change the plant optimum with time (Loeblein \& Perkins, 1999).

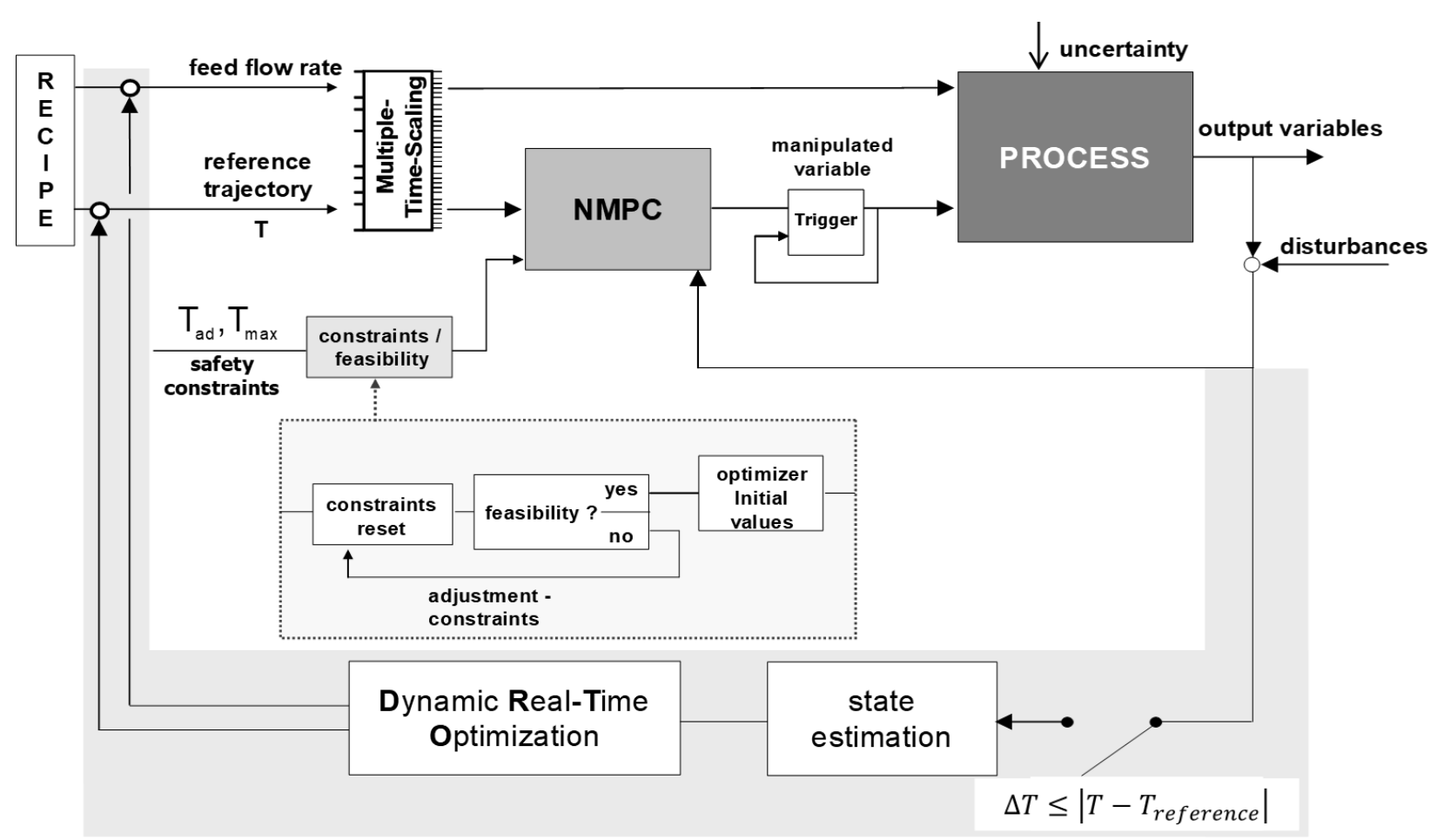

Figure 7: Online framework: Integration of NMPC and dynamic re-optimisation

Thus, a re-optimisation, i.e., dynamic real-time optimisation (D-RTO) may be indispensable for an optimal operation. When on-line measurement gives access to the system state, on-line reoptimisation promises considerable improvement. Moreover, additional constraints can be formulated. In this case study, the state information is assumed to be available and parameters 
are estimated from available measurements. The proposed online framework is illustrated in Figure 7.

This online framework provides a basis for feedback from the process to both the NMPC tracking controller and to the trajectory design level. This also means that the developed two-level strategy for the transient process relies in principle on the assumption that the existing disturbances can be divided into fast and slow time-varying non-zero mean disturbances or drifting parameters.

The update of new trajectories is however performed on a larger time-scale than the sampling time of the controller.

\subsection{Robust chance-constrained NMPC under uncertainty}

Model predictive control has been used extensively in process control engineering. One reason for its popularity is the ability to include directly constraints in the computation of the control moves. However, since the prediction of future process outputs within an NMPC moving horizon is based on a process model involving the effects of manipulated inputs and disturbances on process outputs, the compliance with constraints on process outputs is more challenging than with the ones on process inputs.

Moreover, as the model involves uncertainty, process output predictions are also uncertain. This results in output constraints violation by the closed-loop system, even though predicted outputs over the moving horizon might have been properly constrained. Consequently, a method of incorporating uncertainty explicitly into the output constraints of the online optimisation is needed.
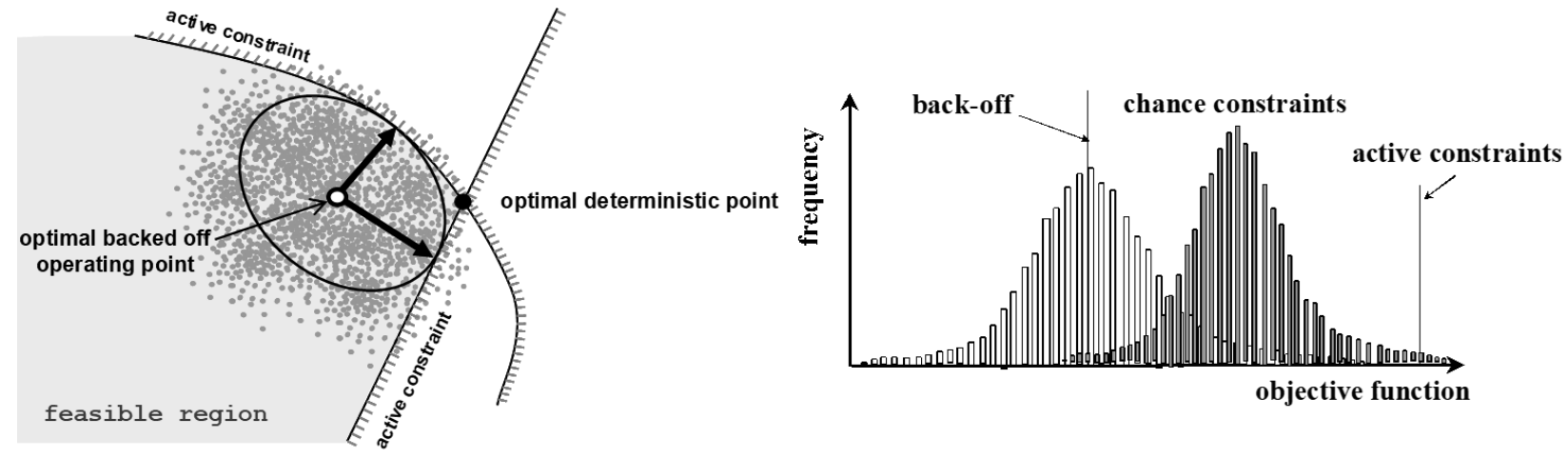

Figure 8: Distribution of the operating points

Furthermore, as discussed in the previous Section, the true process optimum can lie on the boundary of the feasible region defined by one or more active constraints and, thus, it represents a risk of infeasibility at every sampling instant. In addition, the dynamic operating region around the backed-off optimum is certainly not a rigid shape determined by the corresponding back-offs from the safety constraints, but corresponds rather to a distribution of points which are closer to the nominal optimum (see Figure 8 ) and thus leading to a better performance.

Therefore, in this Section, a new robust NMPC scheme is proposed by means of using the chance constrained approach (Li et al., 2008; Arellano-Garcia \& Wozny, 2009). Here, in particular, closed- 
loop stochastic dynamic optimisation problems are solved assuring both robustness and feasibility with respect to output constraints.

The main concept lies in the consideration of unknown and unexpected disturbances in advance. The approach considers the nonlinear relation between the uncertain input and the constrained output variables. The new controller solves a chance-constrained nonlinear dynamic optimisation problem at each execution in order to determine the set of control moves that will optimise the expected performance of the system while complying with the constraints. The controller deals with model uncertainty and disturbances, which are assumed to be correlated multivariate stochastic variables, by replacing the deterministic inequality constraints in the NMPC formulation with chance constraints which are to be complied with a predefined probability level.

The formulation and tuning of individual predefined probability limits of complying with the restrictions incorporate the issue of feasibility, and the consideration of trade-off between the objective function (profitability) and robustness. Thus, the solution of the problem has the features of prediction, robustness and being closed-loop.

\subsubsection{Chance constrained linear MPC}

For linear MPC with single chance constraints, the chance constraints can easily be transformed to linear deterministic inequalities where the uncertain variables in the prediction horizon are described as random variables with a probability distribution function, and the output constraints are formulated as chance constraints. This leads to a QP problem and thus the solution can be derived analytically (Schwarm \& Nikolaou, 1999). For problems with a joint chance constraint, an explicit solution cannot be obtained since the calculation of a joint probability of multivariate uncertain variables is needed. Here, the resulting linear chance constrained MPC problem is then transformed into a convex nonlinear optimisation problem so that it can be solved with a standard NLP method. It should be noted that even if the uncertain inputs are uncorrelated, the outputs are correlated through the linear propagation.

Linear MPC under probabilistic (chance) constraints have been proposed in ( $\mathrm{Li}$ et al., 2000; Wendt, 2005). In these studies, the distribution of disturbances is considered in the design of chance constrained MPC controllers, so that the resulting control performance is more robust than that by the conventional MPC design methods. However, unlike the linear case, for nonlinear (dynamic) processes the controls have also an impact on the covariance of the outputs

\subsubsection{Chance constrained nonlinear MPC}

In this Section, a chance constrained programming framework is used to propose a robust nonlinear model predictive control strategy. The basic idea is to avoid directly computing the output probability distribution. Instead, an equivalent representation of the probability is derived by mapping the probabilistic constrained output region back to a bounded region of the uncertain inputs. Thus, the probability computation of the output constraints is transformed to a multivariate 
integration in the limited area of uncertain inputs (Li et al., 2008; Arellano-Garcia et al., 2009; Barz et al., 2011). Based on the formulation for the tracking controller discussed in Section 3.1, the general chance constrained NMPC problem, which is solved at each sampling time $k$, can be formulated as follows:

$\min _{\dot{V}_{\text {cool }}} \mathcal{J}\left(N_{1}, N_{2}, N_{U}\right)=\sum_{k=N_{1}}^{N_{2}} \delta(k) \cdot[\hat{y}(t+k \mid t)-w(t+k)]^{2}+\sum_{k=N_{1}}^{N_{U}} \lambda(k) \cdot[\Delta u(t+k-1)]^{2}$

s.t.

$x(k+i+1 \mid k)=g_{1}(x(k+i \mid k), u(k+i \mid k), \xi(k+i))$

$y(k+i \mid k)=g_{2}(x(k+i \mid k), u(k+i \mid k), \xi(k+i))$

$\operatorname{Pr}\left\{y_{\min } \leq y(k+i \mid k) \leq y_{\max }\right\} \geq \alpha \quad i=1, \ldots, n$

$u_{\min } \leq u(k+i \mid k) \leq u_{\max } \quad i=0, \ldots, m-1$

$\Delta u_{\min } \leq \Delta u(k+i \mid k)=u(k+i \mid k)-u(k+i-1 \mid k) \leq \Delta u_{\max }$

Where $g_{1}$ are the first-principles model equations describing the dynamic changes of the state variables $x$, while $g_{2}$ describe the state of the constrained variables $y$ depending on the control variables $u$ and the uncertain parameters $\xi$.

The main novelty of the chance constrained NMPC relies on the explicit inclusion of the uncertainties in the problem formulation. The principles of the control strategy are schematically depicted in Figure 9.

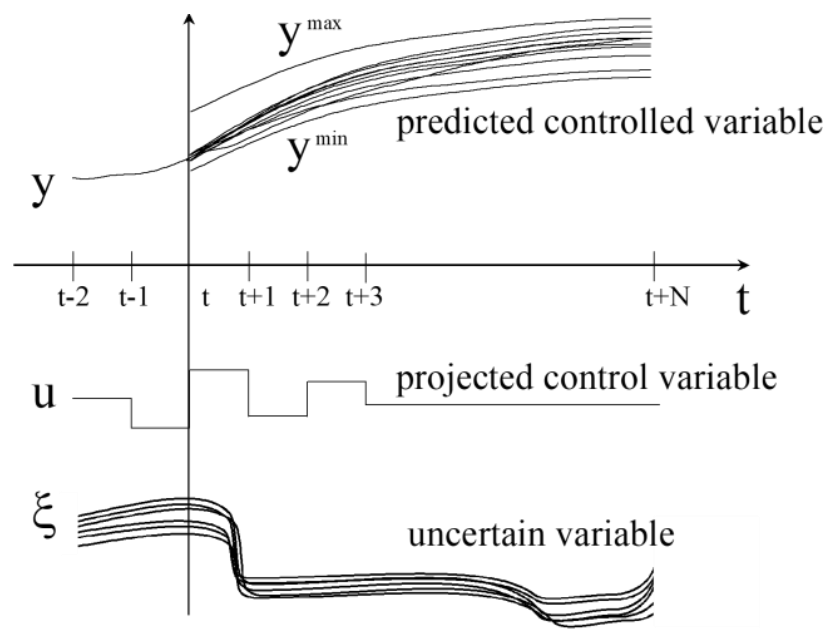

Figure 9: Principles of chance constrained model predictive control

Based on the current output variable $y(t)$ and the input $u(t-1)$ the future $\mathrm{N}$ controls will then be computed such that the predicted outputs are restricted within the specified bandwidth with a given probability. Once the control is implemented including the realisation of the disturbances, the new state at the time-point $t+1$ is accessible. The computation is then repeated in the next horizon. 
The efficiency of the chance-constrained controller is demonstrated through the application to the same case study of the fed-batch reactor under safety constraints discussed throughout this paper. The resulting NMPC scheme is also embedded in the on-line optimisation framework (Figure 7). For the sake of simplicity, the objective function only includes the quadratic terms of the controls, since the outputs are confined by the chance constraints.

Moreover, the relationship between the probability level and the corresponding value of the objective function can also be used here for a suitable trade-off decision between profitability and robustness. As previously discussed, tuning the value of $\alpha$ is also an issue of the relation between feasibility and profitability. However, the general solution of the defined NMPC problem from Equation (2) is only able to arrive at a maximum value $\alpha^{\max }$ which is dependent on the properties of the uncertain inputs and the restriction of the controls. The value of $\alpha^{\text {max }}$ can be computed through a prior probability maximisation step. For this purpose, the following optimisation problem is then solved:

$\max \alpha$

$$
\begin{array}{ll}
\text { s.t. } & g(\dot{x}, x, y, u, \xi)=0, \quad x\left(t_{0}\right)=x_{0} \\
& h_{D}(\dot{x}, x, y, u, \xi) \leq 0 \\
& \operatorname{Pr}\left\{T \leq T_{\max }\right\} \geq \alpha \\
& u_{\text {min }} \leq u \leq u_{\max }
\end{array}
$$

Where $g$ represents the model equations, which form the equality constraints, and $h_{D}$ are the deterministic inequality constraints. The maximisation of $\alpha$ is equivalent to the computation of the highest probability value of complying with the constraint, which can be maximised as an ordinary objective function by setting the optimal values of the decision variables $u$.

\section{Problem formulation}

\subsection{Fed-batch reactor model}

In this case study, a detailed first-principles model of the exothermic fed-batch process is given by a set of DAEs based on:

- Material balances:

$\frac{d n_{A}}{d t}=-v_{A} \cdot k_{01} \cdot e^{\frac{-E_{1}}{R T}} \cdot \frac{n_{A}^{2}}{V}+$ feed

$\frac{d n_{B}}{d t}=-k_{02} \cdot e^{\frac{-E_{2}}{R T}} \cdot n_{B}+k_{01} \cdot e^{\frac{-E_{1}}{R T}} \cdot \frac{n_{A}^{2}}{V}$

$\frac{d n_{C}}{d t}=k_{02} \cdot e^{\frac{-E_{2}}{R T}} n_{B}$

- Reactor energy balance:

$\frac{d}{d t}\left[T \cdot n_{S} \cdot \sum_{i}^{c o m p}\left(c_{p_{i}} \cdot x_{i}\right)\right]=\dot{Q}_{\text {reac }}+\dot{Q}_{\text {feed }}+\dot{Q}_{\text {cool }}^{H T}$ 
- Cooling jacket energy balance:

$\frac{d \bar{T}_{c o o l}}{d t}=\frac{\dot{V}_{c o o l} \cdot \rho_{c o o l} \cdot c_{p, c o o l} \cdot\left(T_{c o o l, i n}-\bar{T}_{c o o l}\right)-\dot{Q}_{c o o l}^{H T}}{V_{c o o l} \cdot \rho_{c o o l} \cdot c_{p, c o o l}}$

- Constitutive algebraic equations

$\dot{Q}_{\text {reac }}=-\sum_{i}^{c o m p}\left(h_{i} \cdot \frac{d n_{i}}{d t}\right)=\sum_{i}^{c o m p}\left[h_{0 i}+c_{p i} \cdot\left(T-T_{0}\right)\right] \cdot \frac{d n_{i}}{d t} \quad i=A, B, C$

$\dot{Q}_{f e e d}=\left[h_{0 i}+c_{p i} \cdot\left(T_{F}-T_{0}\right)\right] \cdot$ feed

$\dot{Q}_{\text {cool }}^{H T}=-k_{H T} \cdot A \cdot\left(T-\bar{T}_{\text {cool }}\right)=-k_{H T} \cdot\left(\frac{\pi \cdot d^{2}}{4}+4 \cdot \frac{V}{d}\right) \cdot\left(T-\bar{T}_{\text {cool }}\right)$

$n_{S}=n_{A}+n_{B}+n_{C}$

$V=\frac{n_{A} \cdot \widetilde{M}_{A}+n_{B} \cdot \widetilde{M}_{B}+n_{B} \cdot \widetilde{M}_{B}}{n_{A} \cdot \widetilde{\widetilde{\rho}}_{A}+n_{B} \cdot \overline{\tilde{\rho}}_{B}+n_{C} \cdot \overline{\widetilde{\rho}}_{C}} \cdot n_{S}$

$n_{S} \cdot \sum_{i}^{c o m p}\left(c_{p_{i}} \cdot x_{i}\right)=c_{p A} \cdot n_{A}+c_{p B} \cdot n_{B}+c_{p C} \cdot n_{C}$

In these equations $V$ denotes the time-varying reactor volume; $n_{i}$ the molar amount of component $i=A, B, C ; T, T_{F}, \bar{T}_{\text {cool }}, T_{\text {cool }}$ the reactor, dosing, jacket and cooling medium temperatures, respectively; $h_{0 i}$ are the specific standard enthalpies; $k_{H T}$ the heat transfer coefficient; $d$ the scaled reactor diameter; $S$ the heat exchanger surface; $\widetilde{M}_{i}$ the molecular weights; $\rho_{i}$ densities; $c_{p i}$ the heat capacities. Additional data and parameters corresponding to the batch reactor are given in Table 1.

Table 1: Process data and parameter of the exothermic batch reactor

\begin{tabular}{|c|c|c|c|c|c|c|c|c|}
\hline Parameter & Unit & Value & Parameter & Unit & Value & Parameter & Unit & Value \\
\hline$v_{A}$ & & 2 & $\rho_{A}$ & $\mathrm{~g} / \mathrm{dm}^{3}$ & 550 & $k_{H T}$ & $\mathrm{~W} / \mathrm{dm}^{2} / \mathrm{K}$ & 10 \\
\hline$T_{0}$ & $\mathrm{~K}$ & 273 & $\rho_{B}$ & $\mathrm{~g} / \mathrm{dm}^{3}$ & 800 & $V_{\text {jacket }}$ & $\mathrm{dm}^{3}$ & 6.98 \\
\hline$R$ & $\mathrm{~J} / \mathrm{mole} / \mathrm{K}$ & 8.31441 & $\rho_{C}$ & $\mathrm{~g} / \mathrm{dm}^{3}$ & 900 & $F e e d$ & $\mathrm{~mole} / \mathrm{s}$ & \\
\hline$c_{p A}$ & $\mathrm{~J} / \mathrm{mole} / \mathrm{K}$ & 92.3 & $k_{01}$ & $1 / \mathrm{s}$ & 500 & $\dot{V}_{\text {cool }}$ & $\mathrm{dm}^{3} / \mathrm{s}$ & \\
\hline$c_{p B}$ & $\mathrm{~J} / \mathrm{mole} / \mathrm{K}$ & 154.2 & $k_{02}$ & $1 / \mathrm{s}$ & 10,000 & $h_{0 A}$ & $\mathrm{~J} / \mathrm{mole}$ & 48,500 \\
\hline$c_{p C}$ & $\mathrm{~J} / \mathrm{mole} / \mathrm{K}$ & 173.9 & $D$ & $\mathrm{dm}$ & 3 & $h_{0 B}$ & $\mathrm{~J} / \mathrm{mole}$ & 36,500 \\
\hline$\widetilde{M}_{A}$ & $\mathrm{~g} / \mathrm{mole}$ & 25 & $\rho_{\text {cool }}$ & $\mathrm{g} / \mathrm{dm}^{3}$ & 900 & $h_{0 C}$ & $\mathrm{~J} / \mathrm{mole}^{2}$ & 30,000 \\
\hline$\widetilde{M}_{B}$ & $\mathrm{~g} / \mathrm{mole}$ & 50 & $c_{p, \text { cool }}$ & $\mathrm{J} / \mathrm{g} / \mathrm{K}$ & 3.1 & $E_{1}$ & $\mathrm{~J} / \mathrm{mole}$ & 48,890 \\
\hline$\widetilde{M}_{C}$ & $\mathrm{~g} / \mathrm{mole}$ & 50 & $T_{\text {cool }}$ & $\mathrm{K}$ & 298 & $E_{2}$ & $\mathrm{~J} / \mathrm{mole}$ & 53,000 \\
\hline
\end{tabular}

The resulting model is comprised of five differential and two algebraic state variables. Moreover, there are three time-varying operational degrees of freedom: the feed flow rate into the reactor, the cooling flow rate, and the length of the different time intervals. 


\subsection{Physical and safety restrictions}

The consideration of safety aspects, in particular physical safety, is essential. They often determine the recipe design procedure and limit the achievable performance. Thus, their consideration is required in order to operate the process closer to the existing constraints. The developed model considers both the reactor and the cooling jacket energy balance. Thus, the dynamic performance between the cooling medium flow rate as manipulated variable and the controlled reactor temperature is also included in the model equations. Thereby, it can be guaranteed that later the computed temperature trajectory can be implemented by the controller. Furthermore, whilst the reaction proceeds, the reactor's volume varies so that the computation of the corresponding cooling capacity is adapted according to the required cooling jacket area. Besides, since the heat removal is limited, the temperature is controlled by the feed rate of reactant $\mathrm{A}$ (semi-batch operation mode), and the flow rate of the cooling liquid, $\dot{V}_{\text {cool }}$. At the start, the reactor partly contains the total available amount of $A$. The remainder is then fed and its feed flow rate is optimised to maximise the yield. However, the accumulation of $A$ at the start of the batch time must be prevented, otherwise, as the batch proceeds exhaustion of the cooling system capacity cannot be avoided.

\subsubsection{Maximal reactor temperature}

In order to guarantee operability within a specified operating regime it should be assured that the occurring heat generated can be discharged through the cooling system at any time. Thus, there exists a maximal allowable reactor temperature starting from which the maximum cooling performance is no longer enough to run the process within the specified product requirements. Hence, there is an upper bound according to the critical reactor temperature which should not be exceeded at any time point during the whole batch process. Consequently, the following condition results:

$\dot{Q}_{\text {reac }}\left(T_{\text {max }}\right) \leq\left|\dot{Q}_{\text {cool }}^{\text {HT, max }}\left(T_{\max }\right)\right|$

This implies that in each time point it must be possible to lower both the reactor temperature and the cooling jacket temperature with the maximum available cooling flow rate in order to fulfil condition (13). This leads to:

$\frac{d T}{d t} \leq 0$ and $\frac{d \bar{T}_{\text {cool }}}{d t} \leq 0$

In this case, the relations given in (14) are inserted in Equations (7) and (8), respectively, to obtain:

$$
\begin{aligned}
& \bar{T}_{\text {cool }}^{\text {min }}=\bar{T}_{\text {cool }} \leq \frac{-\dot{Q}_{\text {reac }}}{k_{H T} \cdot\left(\frac{\pi \cdot d^{2}}{4}+4 \cdot \frac{V}{d}\right)}+T \\
& \bar{V}_{\text {cool }}^{\text {min }} \geq \frac{\dot{Q}_{\text {reac }}}{\rho_{\text {cool }} c_{p, \text { cool }}\left(T_{\text {cool,in }}-\bar{T}_{\text {cool }}\right)}
\end{aligned}
$$


Equation (17) indicates the minimal required cooling jacket temperature in order to comply with condition (16), while Equation (18) denotes the required minimal cooling pump capacity in order to remove the reaction heat. This issue is illustrated in Figure 10.

In particular, Figure 10 shows that once the reactor temperature exceeds $300 \mathrm{~K}$, a sufficiently large gradient exists for heat removal over the cooling jacket. Moreover, the required minimum temperature of the cooling medium in the cooling jacket is higher than the cooling inlet temperature. Additionally, for lower reactor temperatures the necessary pump capacity is rather low, though it escalates for higher reactor temperatures reaching its maximal value of $0.3 \mathrm{l} / \mathrm{s}$ at $365 \mathrm{~K}$, which is predefined by the pump design. Thus, the maximal permitted operating temperature for the reactor can be specified explicitly.

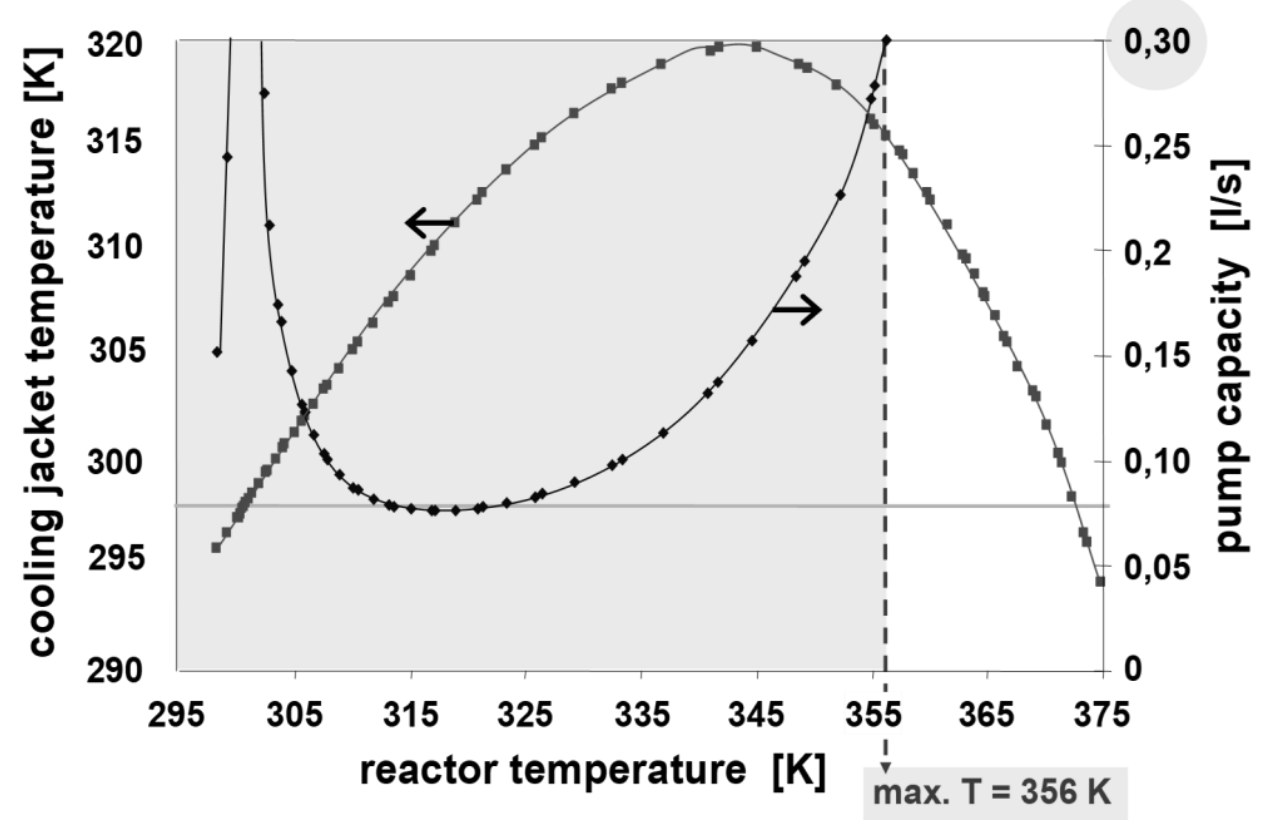

Figure 10: Simulated minimal cooling jacket temperature and cooling pump capacity

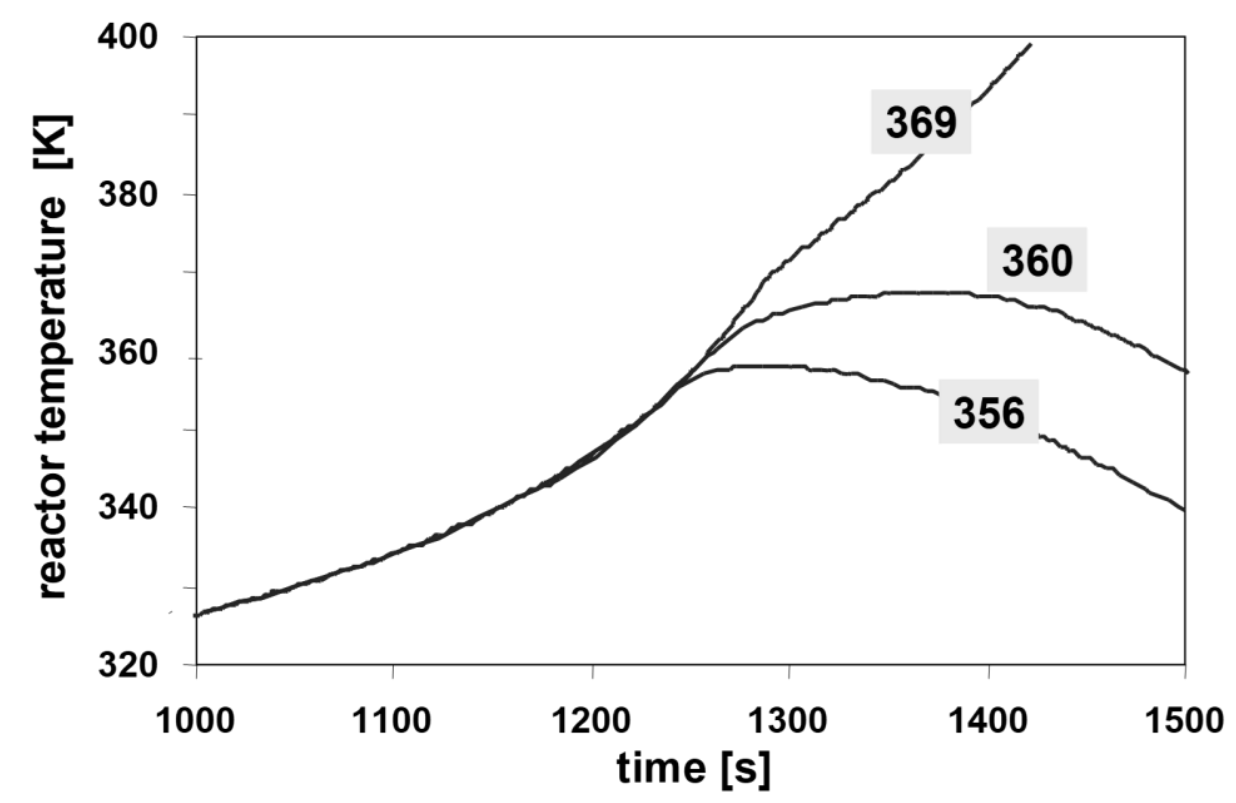

Figure 11: Simulated minimal cooling jacket temperature and cooling pump capacity 
The results in Figure 11 correspond to the case when the reactor is charged with the entire amount of reactant $A$ and the cooling system is switched off. As soon as the reactor temperature reaches a certain temperature, the cooling pump will be put into operation at full capacity.

In the first case $(T=356 \mathrm{~K})$ represented in Figure 11, it can be seen that the reactor temperature can be lowered with a slight delay, and it achieves the maximum value of $358 \mathrm{~K}$. On the other hand, if the pump starts up when the reactor temperature is at $360 \mathrm{~K}$, a further temperature rise cannot be stopped and the reactor is no longer controllable. Finally, a runaway cannot be avoided by switching on the pump at $369 \mathrm{~K}$.

\subsubsection{Safety restrictions to avoid runaways}

The operation of the fed-batch reactor requires not only operability within specified operating regimes but also in non-specified regimes. Since the reaction is strongly exothermic, great care is necessary in order to ensure that the determined operation remains safe. In this study, the particular scenario of a sudden cooling failure is taken into account. However, since the time instant of a possible failure is not known a priori, a safety constraint is incorporated which has to be satisfied during the whole operating path. This implies verification through dynamic simulation at every time point. Thus, based on the thermal explosion theory used for the analysis and design of reaction processes under safety considerations, the adiabatic temperature rise, $\Delta T_{a d}$ is considered to deal with the dynamics of the cooling system failure (Abel et al., 2000). However, since the maximum achievable temperature after failure is primarily of interest at any time point, once a runaway has started, it can be assumed that the feed is stopped immediately and that the reaction will carry on under adiabatic conditions. Consequently, the heat produced by the reaction will cause a temperature rise whose extent depends on the momentary concentrations, reactor temperature, cooling jacket temperature, the heat capacity of the reactor content, as well as on the reactor heat generation. This can be calculated by a stationary energy balance around the reactor assuming adiabatic conditions. Thus, with the reactor temperature, $T$, in progress, the adiabatic end temperature, $T_{a d}$ can then be described as follows:

$T_{a d}=T(t)+\Delta T_{a d}$

It results that:

$T_{a d}=\frac{\sum_{i}^{\operatorname{comp}}\left[h_{0 i}+c_{p i} \cdot\left(T-T_{0}\right)\right]}{c_{p C} \cdot\left(n_{C}+\frac{n_{A}}{2}+n_{B}\right)+V_{\text {cool }} \cdot \rho_{\text {cool }} \cdot c_{p, \text { cool }}}+\frac{V_{\text {cool }} \cdot \rho_{\text {cool }} \cdot c_{p, \text { cool }} \cdot T_{\text {cool }}+\left(n_{C}+\frac{n_{A}}{2}+n_{B}\right) \cdot\left(c_{p C} \cdot T_{0}-h_{0 C}\right)}{c_{p C} \cdot\left(n_{C}+\frac{n_{A}}{2}+n_{B}\right)+V_{\text {cool }} \cdot \rho_{\text {cool }} \cdot c_{p, \text { cool }}}$

Hence, Equation (20) represents a further algebraic relation which can be used also to restrict the variable $T_{a d}$ by a temperature limit converting the dynamic treatment of the cooling system failure to a path constraint. In order to emphasise this issue, the development of the reactor temperature, the cooling jacket temperature as well as the reaction performance are illustrated in Figure 12 considering the current feed amount in the reactor (500 moles of reactant $A$ ) and a switched-off cooling. 
Due to the stationary balance, the instantaneous increase of the temperature is not covered. The heat produced, however, is transferred relatively fast to the cooling jacket reaching temperature compensation.

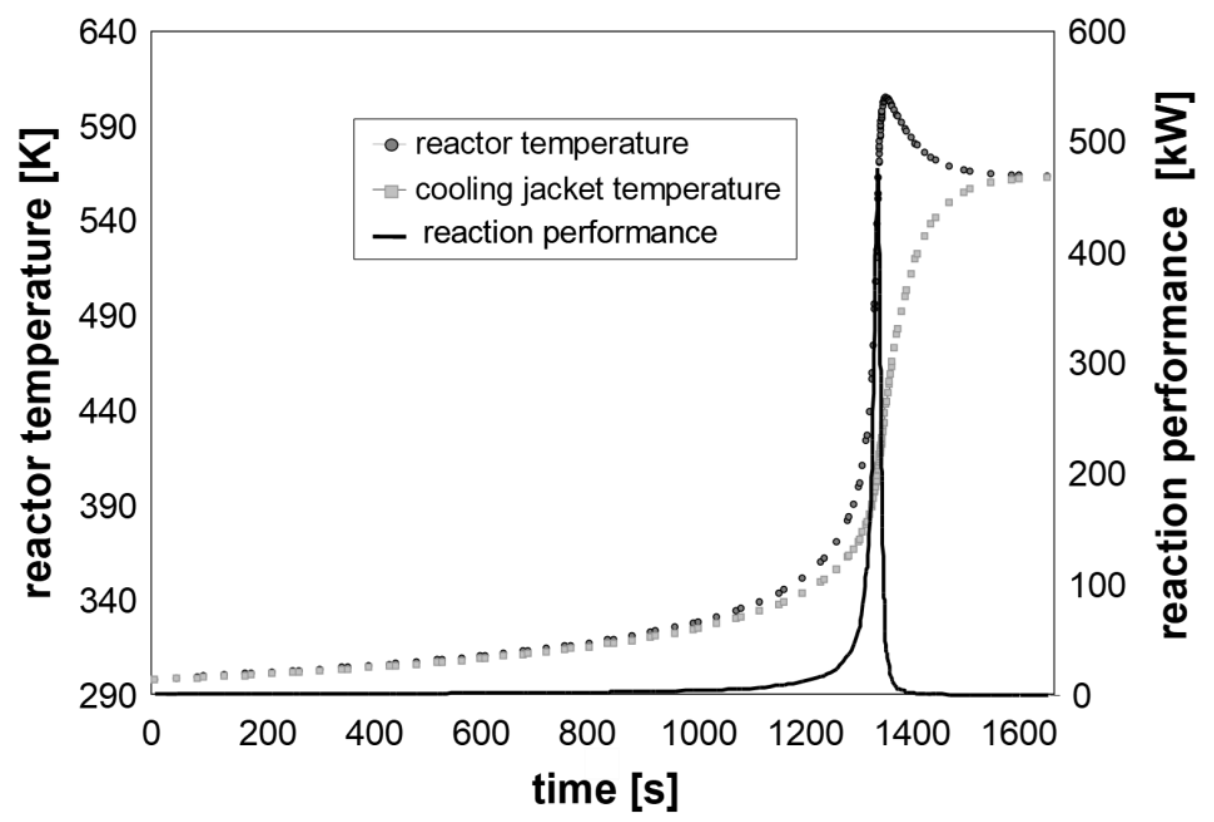

Figure 12: Batch heating-up procedure with switched-off cooling

Furthermore, the maximum possible temperature without heat dissipation to the environment is $683 \mathrm{~K}$. Because of the heat transfer to the cooling jacket, the adiabatic end temperature of the reactor content plus cooling jacket is $564 \mathrm{~K}$. On the other hand, if the initial feed amount of reactant $\mathrm{A}$ is reduced, for instance to 240 moles, then the end temperature will be decreased to $497 \mathrm{~K}$. Thereby, it becomes clear that supplying reactant A during the batch is the most appropriate operation mode in order to comply with the safety constraints because of its sensitivity to the adiabatic end temperature. By this means, the initial amount of reactant $A$ represents also an additional degree of freedom. 


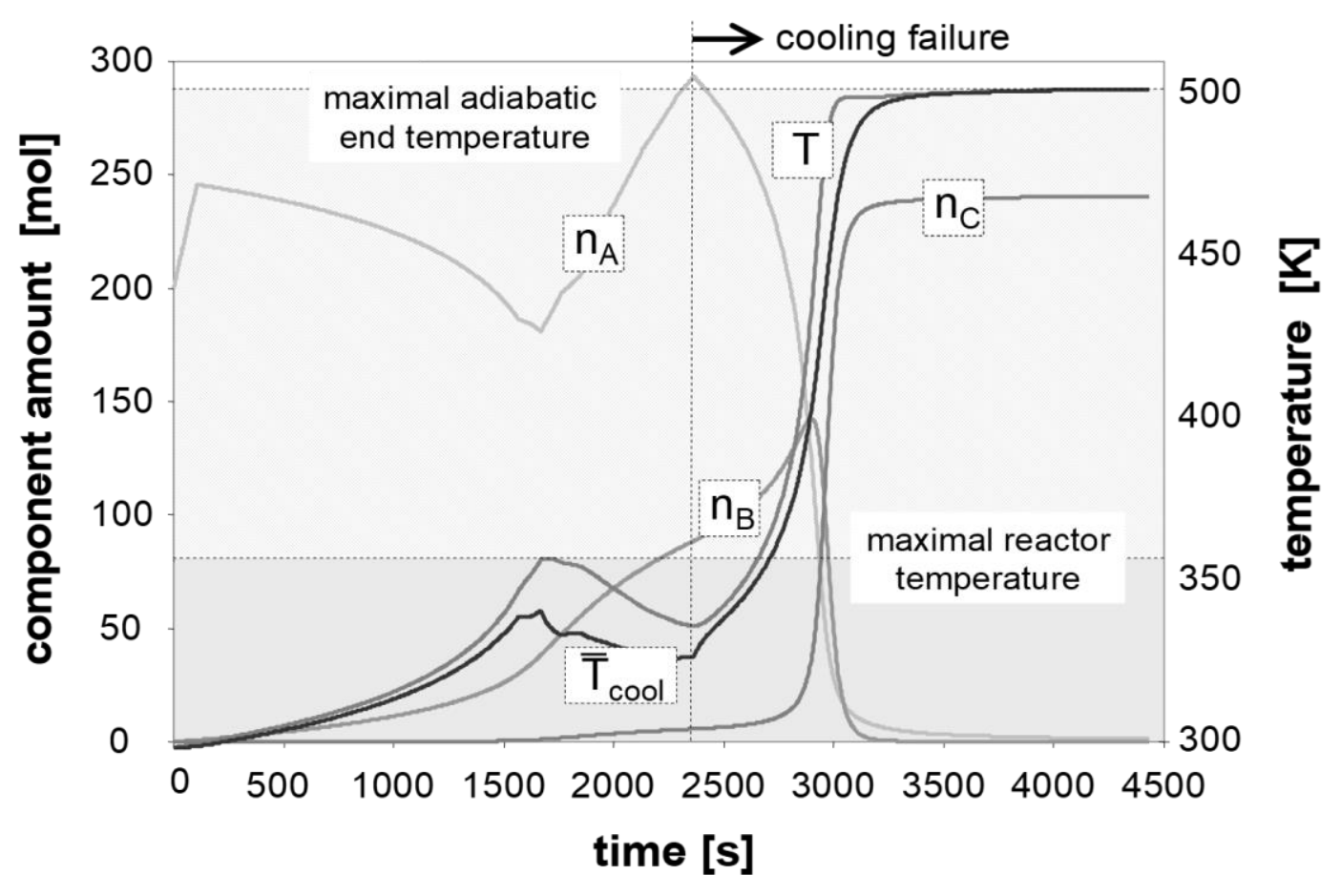

Figure 13: Simulation of cooling failure

Thus, Equation (20) represents a regulation for the reactor operation. If this regulation/constraint is complied with, it can be then guaranteed that in case of a cooling failure, the reactor will not exceed a certain stationary final temperature at any time point. Figure 13 shows the simulation results for $T_{a d}=500 \mathrm{~K}$ and a cooling failure after 2,364 seconds with subsequent switch-off of the cooling pump.

This implies that the limitations of the cooling system (pump capacity) can be taken into account explicitly for the optimisation. In addition, both maximum temperature constraints have to be enforced during the complete batch time. The selected cooling medium is Aral Farolin $U$, which can be used for a temperature range from -10 to $320^{\circ} \mathrm{C}$. Therefore, it does not represent a source of danger in non-specified operating regimes.

\subsection{Optimisation problem formulation}

The optimal operational strategy for the semi-batch reactor is now to be calculated such that the physical and safety restrictions derived in the previous Section are also considered. Moreover, the open-loop optimal control problem needs to be solved first, and represents a prerequisite for the consecutive optimisation with moving horizons involved in NMPC. The objective function is chosen depending on the nature of the problem. Thus, there are, in general, two practical optimisation problems related to batch operation: maximisation of product concentration in a fixed batch time, or minimisation of batch operation time subject to end point constraints in order to determine an optimal reactor temperature profile.

The first problem formulation is applied to a situation where the increase of the desired product amount is required while the batch operation time is fixed. This is due to the limitation of complete 
production line in a sequential processing. However, in some circumstances, we need to reduce the duration of batch run to allow the operation of more runs per day. This requirement leads to the minimum time optimisation problem.

In this problem definition, both issues are combined, thus, the objective is to maximise the production of compound $\mathrm{B}$ at the end of the batch $\left(C B_{f}\right)$ while reducing the total batch time, $t_{f}$ :

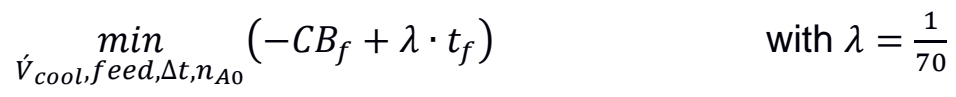

subject to the equality constraints, i.e. process model Equations (4) - (14), as well as path and end point constraints, as outlined immediately next. The numbers in brackets [.] to the left denote the current number of constraints. The parameter $\lambda$ is a weighting factor between the two terms (production of compound $B$ at the end of the batch and the total batch time), whose value was determined through trial-and-error simulations.

First, a limited available amount of reactant $A$, which is to be converted by the final time, is fixed to:

$$
n_{A, t o t a l}\left(t_{f}\right)=n_{A 0}+\int_{t_{0}=0}^{t_{f}} n_{A}(t) d t=500 \text { mole }
$$

At the final batch time, the reactor temperature must not exceed a limit in order to include also the shut-down procedure:

$$
T\left(t_{f}\right) \leq 303 \mathrm{~K}
$$

The safety restrictions are defined as path constraints. The adiabatic end temperature is used amongst others to determine the temperature after failure, as indicator for the educt accumulation, as operation mode regulation, and as process monitoring aid. Handling this constraint ensures that even in the extreme case of a total cooling failure no runaway will occur.

$$
\begin{array}{ll}
\text { [3-42] } & T(t) \leq 356 \mathrm{~K} \\
\text { [43-82] } & T_{a d}(t) \leq 500 \mathrm{~K}
\end{array}
$$

In addition, in order to prevent too large fluctuations of the control variables, the feed and cooling flow rate changes from time interval to interval are restricted to an upper bound. This has a positive effect on the convergence of the optimisation problem, particularly with regard to the initial values for the optimisation run. However, these limitations can also be modified during the optimisation run. For instance, close to the end of the batch process only the absolute amount of heat discharged is of interest for the maximisation of product $B$. Thus, the allowed changes of the cooling flow rate between the neighbouring time intervals are smaller.

[83-122] $\quad\left\|\Delta \dot{V}_{\text {cool }}\right\| \leq 0.05$

[123-162] $\quad \| \Delta$ feed $\| \leq 0.2$ 
The optimisation problem definition includes also the vector of states with known initial condition, $x_{0}$. The decision variables are the feed flow rate into the reactor $(0-3 \mathrm{~mole} / \mathrm{s})$, the cooling flow rate $(0-0.3 \mathrm{l} / \mathrm{s})$, the length of the different time intervals (with values between 60 and 180 seconds) as well as the initial amount of $\mathrm{A}, n_{A 0}$, in the reactor.

The dynamic optimisation problem is solved using the sequential approach, where the variables space is divided into state $x$ and control space $u$. Therefore, only the control or independent variables are optimised by the NLP solver (e.g. SQP). The large-scale DAE system is discretised with the orthogonal collocation on finite elements, thus, the differential and algebraic (dependent) variables are solved throughout the integration of the DAEs with the Newton method, and the required sensitivities computed based on the interval gradient information (Li et al., 1998). The control variables are set as piecewise constant.

The problem is solved assuming 40 time intervals, which results in 121 decision variables for the entire optimisation horizon.

\subsection{Optimal nominal solution}

In this Section, the open-loop optimal results are presented. In the nominal optimisation the uncertainty is ignored. Figure 14 shows the resulting nominal trajectories of the feed and cooling flow rate. During the first few time intervals, the feed flow rate is determined at higher values such that the constraints (24) and (25) are not violated. After a certain feed amount has been added, the feed flow rate is drastically reduced. This ensures a fast ignition of the reaction and a quick conversion.

As soon as the reactor temperature is about to reach its limit value the cooling and, in particular the feed flow rate are again increased. At this time the conversion is sufficiently fast to prevent the accumulation of A. After 2,500 seconds, when the total feed amount has been supplied, the reactor temperature increases rapidly again and arrives at its maximum allowable value where it evolves along its upper limit during a long time period (Figure 15). Subsequently, in order to comply with the shut-down end-point constraint (23), the cooling rate is increased progressively.

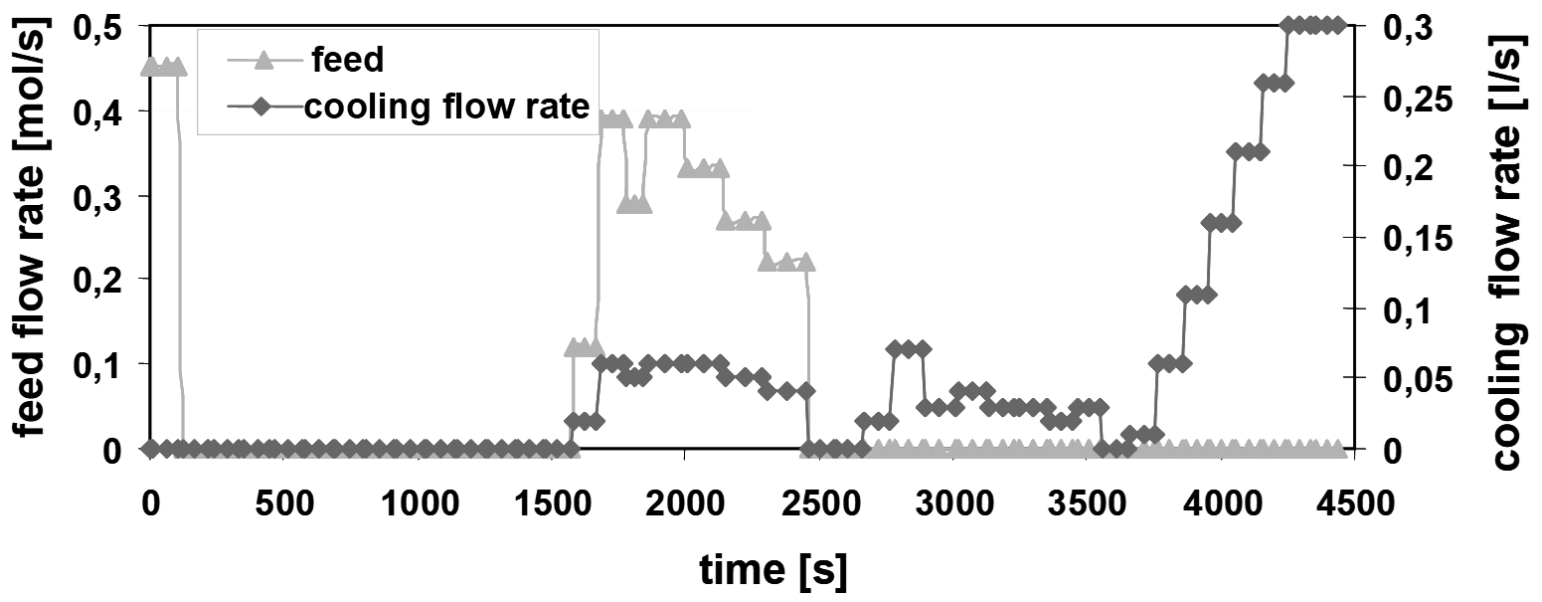

Figure 14: Optimal nominal trajectories of the feed and cooling flow rates 


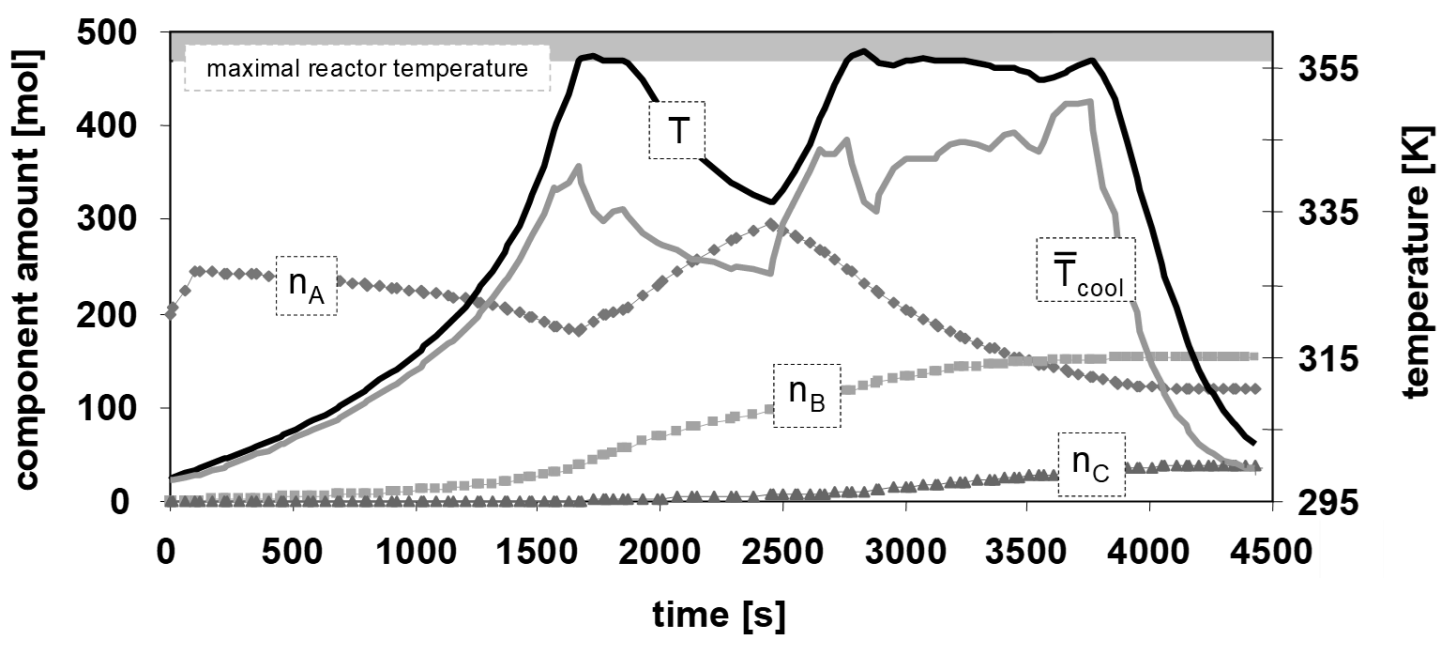

Figure 15: Optimal profiles of the components amount, the reactor temperature and the cooling jacket temperature at nominal optimum

The time-dependent amount of different components, the reactor as well as the cooling jacket temperature are depicted in Figure 16.

From Figures 15 and 16, it can be seen that the safety restrictions and the feed flow rate determine the reactor temperature evolution. Furthermore, the adiabatic end temperature is decisive during the first half of the process run, while in the second part the process is operated almost with maximum reactor temperature until the shut-down period starts. The change of the different time interval lengths can also be noticed, as illustrated in Figure 14. The sum of all 40 time intervals represents the total time. The duration of each individual interval has however a detrimental effect on the applied operational strategy.

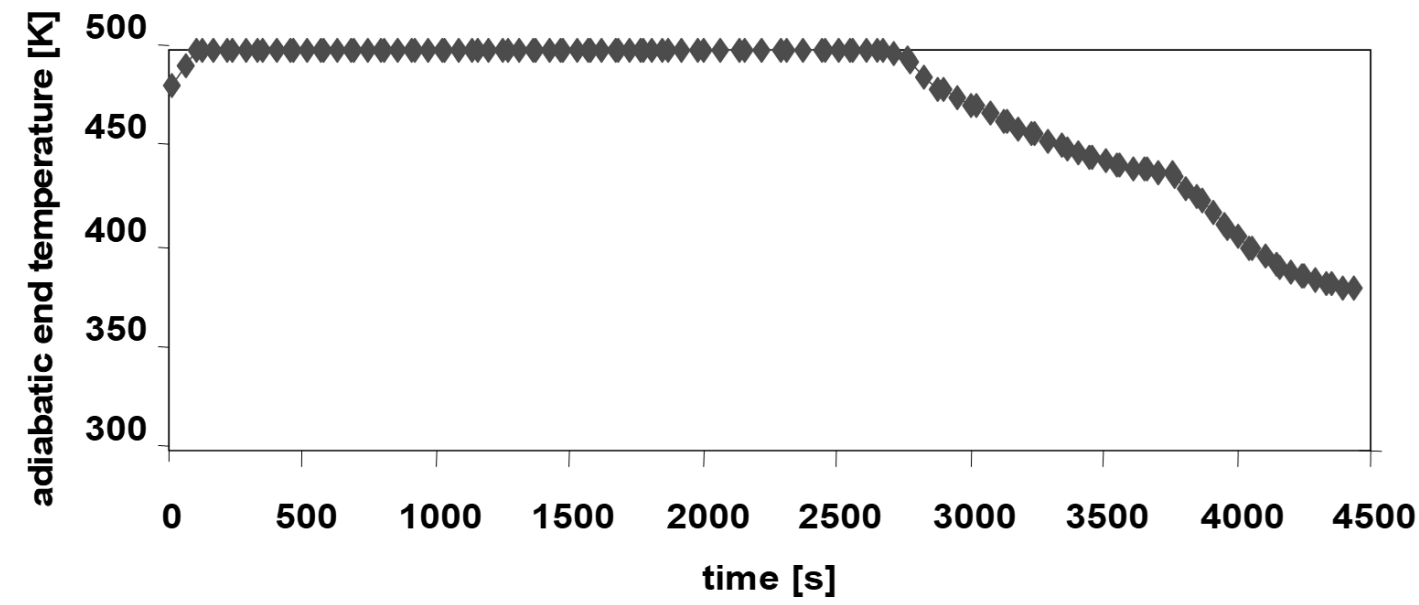

Figure 16: Trajectory of the adiabatic end temperature at nominal optimum

The evolution of the adiabatic end temperature for the nominal operation is depicted in Figure 16. It can be seen that the adiabatic end temperature reaches its upper limit right from the start and remains at this value during the heating process with switched-off cooling (up to 1,500 seconds) and maintains this value through a large part of the batch time. By this means, the reactor temperature and, in particular, the adiabatic end temperature is an active constraint over a large 
time period. This poses a potential risk of violation when uncertainty is involved: although operation at this nominal optimum is desired, it typically cannot be achieved with simultaneous satisfaction of all constraints due to the influence of uncertainties and/or external disturbances. However, the safety constraints should not be violated at any time point. Thus, in this section, two methods based on a NMPC scheme are proposed to implement such an optimal strategy despite disturbances solving closed-loop dynamic optimisation problems assuring robustness with respect to state output constraints within an online framework.

\subsection{NMPC simulation results}

Batch processing offers greater flexibility in the production of speciality and pharmaceutical chemicals. Thus, the trend in the chemical industry towards high added value products has increased interest in the optimal model-based control of batch processes (Bonvin \& François, 2017). Due to its ability to include directly constraints in the computation of the control moves, NMPC presents major advantages for the optimal operation of transient chemical plants. Moreover, it provides a systematic methodology to handle constraints on manipulated and controlled variables, and is not being limited to a certain model structure (Allgöwer et al., 1999). The principle of MPC is shown in Figure 17. The model predictive controller uses a process model to predict the future, and then computes the future control trajectory that optimises a performance objective based on the sum of squares of the differences between model predicted outputs and a desired output variable trajectory over a prediction horizon solving an open-loop optimisation problem.

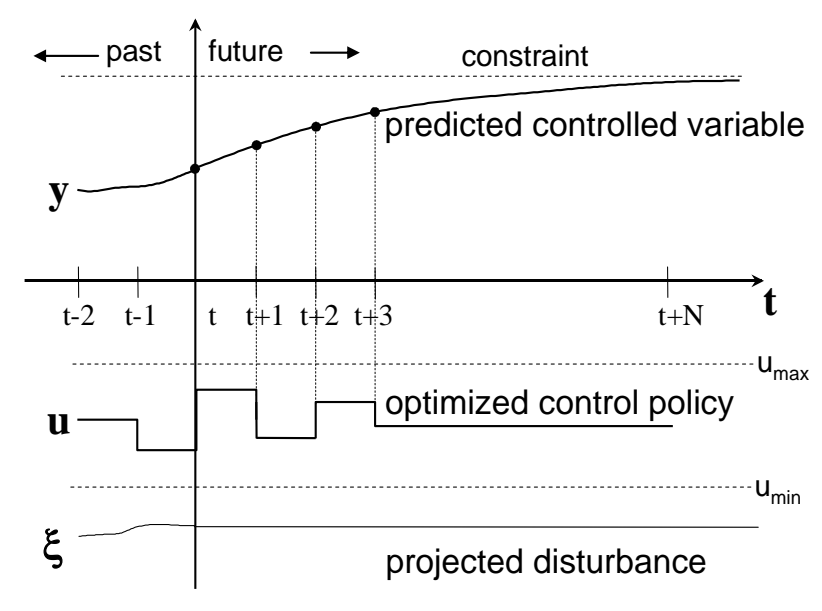

Figure 17: Principles of model predictive control

The value for the control move at the current sampling instance is implemented, i.e. the manipulated variables of the first time interval are applied. At the next sampling instance, new measurements are collected and the control calculation is repeated. Thus, the time window is shifted into the future where the whole procedure is repeated (Figure 18). These steps update the control move calculations to consider the latest measurement information. However, most of the research efforts have mainly been directed towards the regulation problem for stationary 
problems. Recently, a growing number of works have studied the application of MPC to batch and semi-batch processes.

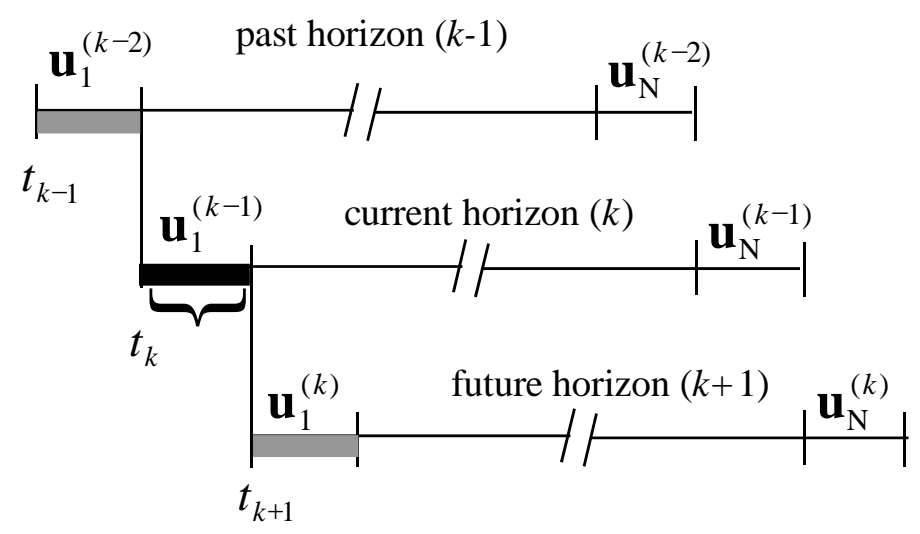

Figure 18: Close-loop optimisation with the moving horizon

However, most of the processes are nonlinear and while linear models are good approximations if the process is kept close to an operating point, this is not the case when the process changes operating point or is subject to large perturbations (Camacho \& Bordons, 1999).

\subsubsection{Open-loop strategy implementation}

Based on the open-loop optimal control trajectories of the critical state variables (see Section 4.4), in this Section, a deterministic NMPC scheme is implemented for the exothermic fed-batch process. In order to implement the open-loop strategy, the 40 intervals from the open-loop optimisation are divided into small intervals with the same length. This is accomplished by a developed multiple-time-scaling strategy which is based on the orthogonal collocation method over finite elements in time. In this strategy, the large time intervals are to be long enough for the practical realisation, as well as for the reduction of the computation time in the sensitivity calculation for the optimisation. In order to keep the continuity of the variables, the last collocation point is used as the starting point of the next interval. Furthermore, small time intervals are adjusted in the simulation and their length is kept more flexible to guarantee the convergence in the Newton iteration. In case of non-convergence, a step length adjustment will be activated to reduce the step length until convergence is achieved. Moreover, the last collocation point of a small time interval must be one of the collocation points of the large time interval. Simulation studies have shown that one advantage of the collocation method is that the solutions of state variables at the same time point are almost independent of the step length, therefore, the state variables of those large intervals can be used to compute the sensitivities. Thus, the gradient calculation has to be done only at the end of one large time interval. As a result, both the number of decision variables and the computation time for the sensitivity calculation can be significantly reduced.

The time length values for these intervals with regard to the decision variables are taken from the off-line optimisation results. The strategy is applied for both discretisation and implementation of 
the optimal policies according to the controller's discrete time intervals (6 - 12 seconds), hence the length will not be lower than 6 seconds. Thereby, applying this procedure will result in a number of around to $600-700$ intervals.

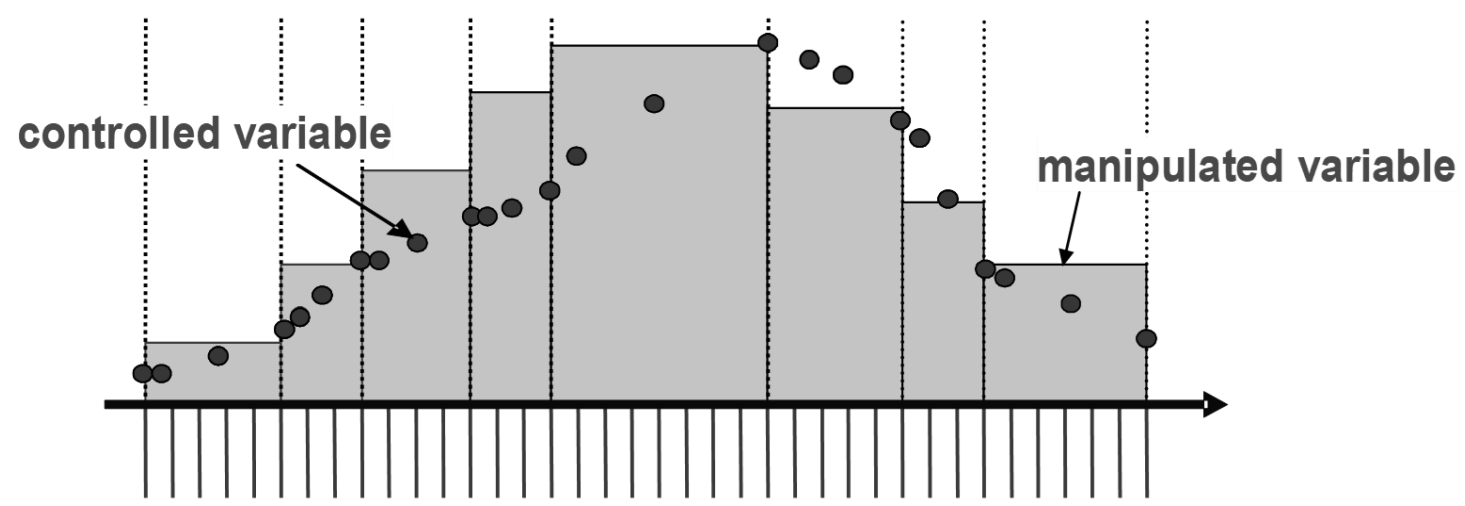

Figure 19: Division of the time interval length based on the multiple-time-scaling approach

In Figure 19, the approach is illustrated systematically. In contrast, the values of the temperature for the beginning of each new interval are interpolated linearly from the previous values of the offline optimal trajectory.

In Figure 20, the open-loop strategy implementation according to the feedforward strategy is presented, i.e. the reactor is operated with the optimal trajectories of the feed and cooling flow rate determined by the off-line deterministic optimisation. However, during the course of a typical batch, process variables swing over wide ranges of values, and process dynamics go through significant changes due to nonlinearity. Furthermore, batch processes are characterised by significant uncertainties, a certain number of noisy measurements, and the fact that the controlled properties are usually not measured on-line.

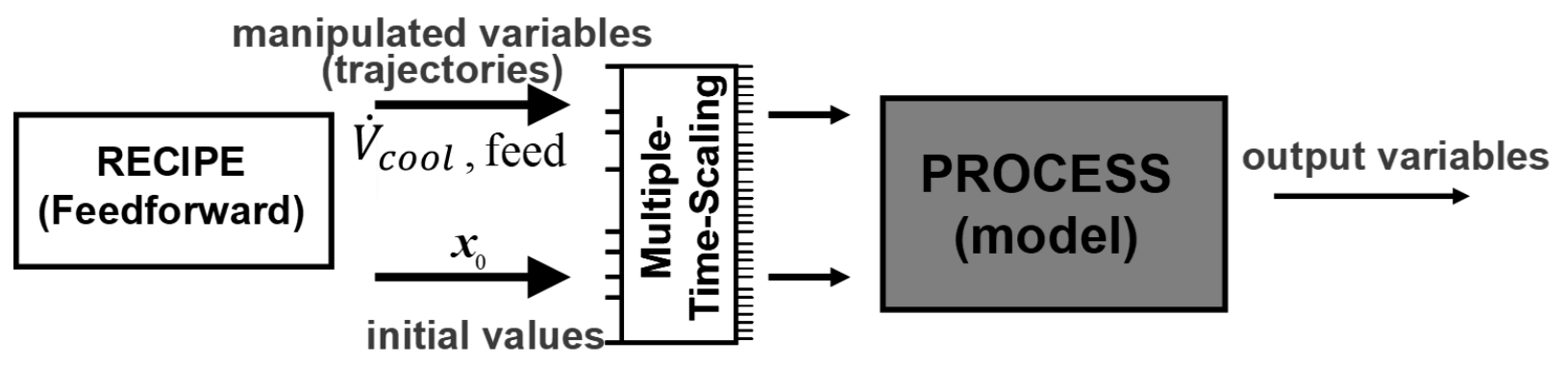

Figure 20: NMPC scheme for the tracking control problem

Thus, in order to show the sensitivity to disturbances the model described in Section 4.1 is extended to include also the catalyst activity, $a$. Thereby, a further factor of influence on the system is realised. For this purpose, a simple approach is used to describe a homogeneous distributed catalyst in the system considered. The catalyst is assumed to influence the main reaction $2 A \rightarrow B$, primarily. So far it was assumed that the total amount of reactant $\mathrm{A}$ is available for the reaction, this is however not the case if the reaction is catalysed. In fact, using a catalyst typically assumes a decrease of the catalyst activity with usage, and therefore a limited conversion of the available educt $A$. This is mainly attributed to the fact that the reaction takes 
place on the catalyst surface and this is not fully available in the course of the reaction any longer. In a simplified form, the catalyst activity can be described as a relationship between the current reaction rate and the reaction rate with the fresh catalyst $(t=0)$. However, if the deactivation mechanism is more complex, the catalyst deactivation kinetics can then not be described independently from the reaction kinetics (non-separable kinetics). In this case study, the catalyst activity is considered directly as a factor of the reaction term in the component balances. The reaction term of the reaction $A$ to $B$ results in:

$r_{A-B}^{\prime}=-a(t) \cdot k(T) \cdot c_{A}$

The kinetics of the catalyst activity is described as follows:

$\frac{d a_{A-B}}{d t}=-K_{\text {decay }} \cdot\left(a_{A-B}\right)^{2} \cdot \frac{n_{A}}{V}$

Where $K_{\text {decay }}$ and $a_{A-B}$ denote the catalyst decay rate and the catalyst diminution, respectively (second order) (Fogler, 1999).

Furthermore, the inlet temperature of the cooling medium into the cooling jacket has been assumed so far to be a constant parameter during the off-line optimisation. However, due to weather or heat input through the cooling pump depending on its momentary performance, the inlet temperature is rather subject to fluctuations. The impact of these changes on the feedforward strategy is depicted in Figure 21, which illustrates the simulation results of the batch operation for an increment of only $2 \mathrm{~K}$ with respect to the cooling inlet temperature from originally $298 \mathrm{~K}$ to 300 $\mathrm{K}$.

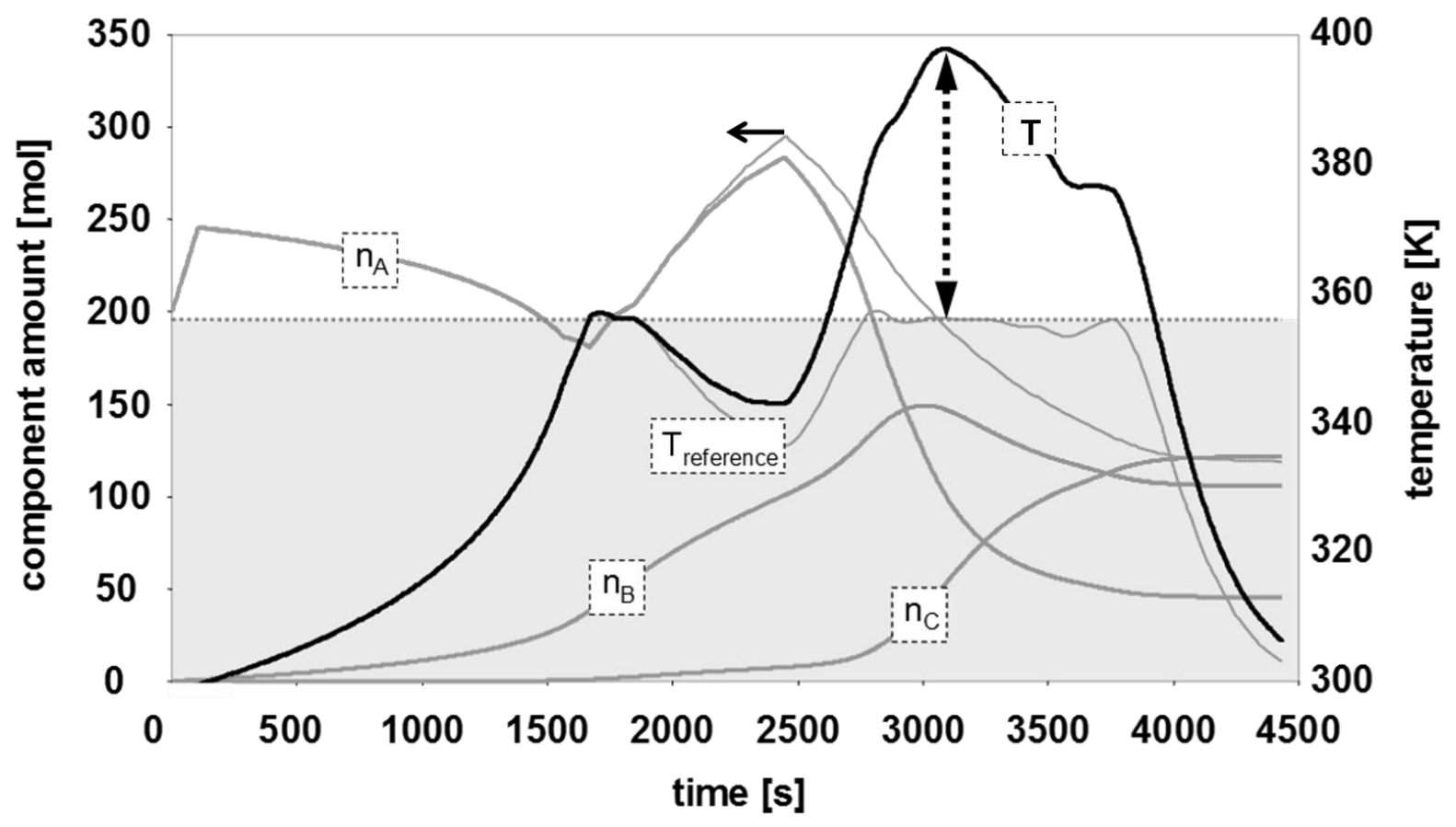

Figure 21: Simulation results of the open-loop strategy implementation with $\Delta T_{\text {cool,in }}=2 \mathrm{~K}$

Here it becomes obvious that the operating conditions deviate significantly from the nominal optimal conditions determined in the open-loop optimisation. In particular, the reference 
trajectories of the educt $A$ in the reactor and the reactor temperature at each time point differ from the simulated trajectories. This has evidently an effect on the yield. However, although operation at the nominal optimum is desired, it typically cannot be achieved with simultaneous satisfaction of all the constraints involved, because of the influence of external disturbances (Loeblein \& Perkins, 1999). Thus, an NMPC based approach is proposed to implement such an optimal strategy despite the presence of disturbances.

\subsubsection{Closed-loop optimisation}

In this Section, a nonlinear model predictive controller is implemented which assures the compliance with the operating conditions tracking the path of the reference trajectory. The feed flow rate control is not included in the closed-loop. Based on simulation studies, the reactor temperature is extremely sensitive to model uncertainty and disturbances. Its impact on the reaction rate is crucial for the course and conversion of the reaction. Thus, the moving horizon tracking controller will then increase the system robustness against external disturbances at some extent.

The process/plant model, is discretised using the orthogonal collocation method with three points per finite element in time. The model includes now the following disturbances: the catalyst decay and the fluctuations of the inlet cooling temperature, which are assumed to be constant parameters within the NMPC process model. The NMPC process model is discretised with the implicit Euler method. Due to the short horizon and the small intervals, both large deviation and error reproduction are not expected. Thus, with constant step length, the computation time can be reduced. This is particularly important with regard to the simulation and the gradient computation for the optimisation. It should be noted that the time required for the solution of the optimisation problem is directly proportional to the interval length and the number of intervals within the moving horizon.

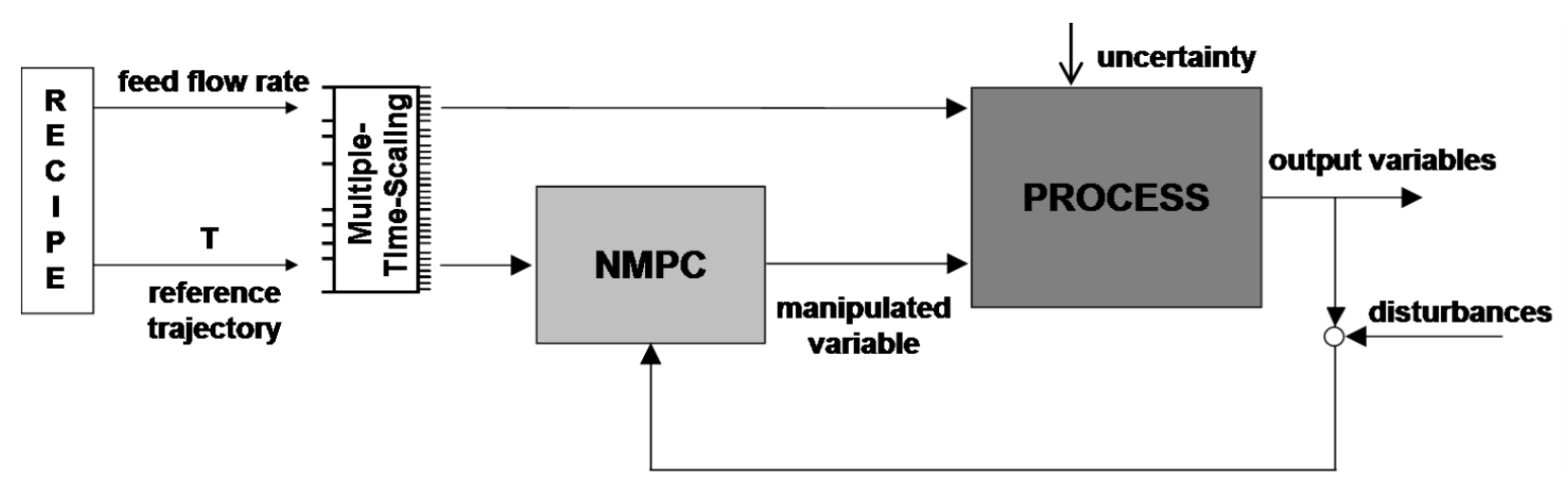

Figure 22: NMPC scheme for the tracking control problem

As shown in Figure 22, in order to implement the developed recipe, the length of the diverse time intervals are first adjusted through the multiple-time-scaling approach. The NMPC controller receives then the discrete set-point trajectory determined via open-loop optimisation. However, the performance of the model based control approach relies on the proper estimation of current 
and future states. Thus, at the end of each corresponding time interval and in time-discrete distance the controller is updated with the current process state. This occurs through measurements at the beginning of each interval (Figure 23).

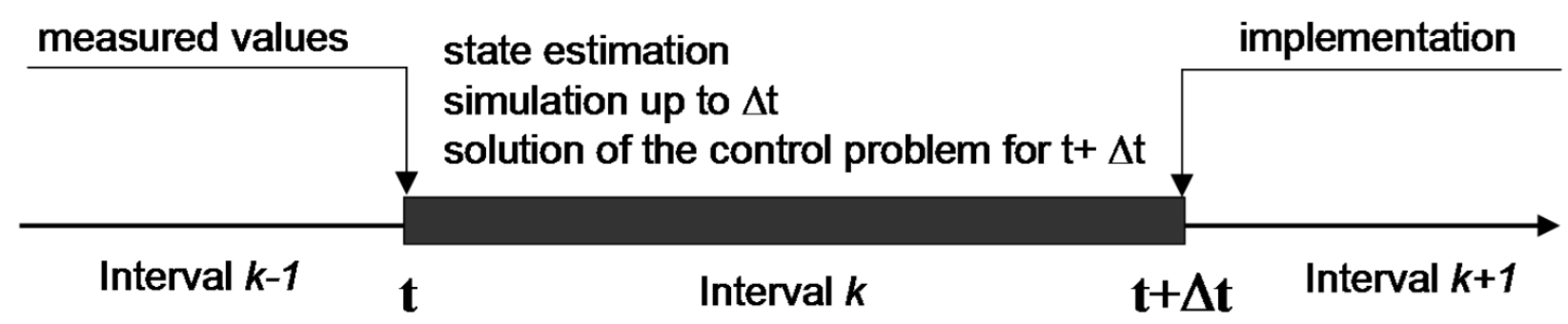

Figure 23: Tasks during the current interval

For this purpose, the values of the components' amounts, and the reactor and cooling jacket temperatures are required in order to describe fully the process state. Since the measurements (here the current values from the process simulation) can commonly not be measured accurately, in this case study these values are corrupted with white noise which for the component amount is $8 \%$ and for the temperature is $2 \%$ of their mean (nominal) values. Subsequently, they are smoothed with a first order filter. By this means, fast disturbances can efficiently be rejected by the controller.

In case the available time within the interval is not sufficient to solve the NMPC problem, a trigger is activated that holds the current control signal for the next interval. Furthermore, as stated before, proper state estimation is crucial for the success of the NMPC application. Extended Kalman filter (EKF) has been widely used in process control applications, however its performance strongly depends on the accuracy of the model (Nagy \& Braatz, 2003; 2004). To avoid highly biased model predictions, some of the model parameters are estimated together with the states. In this case study, the state information is however assumed to be available. For further details concerning the state estimation, we refer to the paper of Haseltine \& Rawlings (2005).

\subsubsection{Tracking problem without safety restrictions}

For the online optimisation of the semi-batch process, the momentary criteria on the restricted controller horizon with regard to the entire batch operation are insufficient. Therefore, the original objective (19) must be substituted by an appropriate alternative that can be evaluated on the local NMPC prediction horizon:

$\min _{\dot{V}_{\text {cool }}} \mathcal{J}\left(N_{1}, N_{2}, N_{U}\right)=\sum_{j=N_{1}}^{N_{2}} \delta(j) \cdot[\hat{y}(t+j \mid t)-w(t+j)]^{2}+\sum_{j=N_{1}}^{N_{U}} \lambda(j) \cdot[\Delta u(t+j-1)]^{2}$

Accordingly, the cooling flow rate becomes now a manipulated variable while the resulting timevariant reactor temperature is taken as reference trajectory. In Equation (30), the first summation of the function stands for the task of keeping as close as possible to the calculated open loop optimal trajectory of the critical variables $\hat{y}$ (e.g., the reactor temperature, which can be measured easily online), whereas the second summation term corresponds to control activity under the 
consideration of the systems' restrictions described above in Equations (22) - (27). Moreover, the control and prediction horizons are chosen equal, to avoid large deviations of the predicted quantities from their set-points due to the transient character of the process. $N_{1}$ and $N_{2}$ denote the number of past and future time intervals, respectively, while $N_{U}$ stands for the number of controls. Both the prediction $T_{P}$ and the control horizon $T_{C}$ are comprised of 8 intervals. The corresponding parameters of the objective function are given in Table 2.

Table 2: Objective function parameters

\begin{tabular}{lll}
\hline$T_{P}$ & prediction horizon & 8 intervals \\
$T_{C}$ & control horizon & 8 intervals \\
$\lambda$ & MV variation weighting factor & 3000 \\
$\delta$ & offset weighting factor & $\alpha^{(T p-j)}$ with $\alpha=0.7$
\end{tabular}

The parameters of the objective function in Table 2 are determined in such a way that the best possible control quality can be achieved compensating for disturbances and model uncertainties. Thus, in order to examine the robustness of the developed strategies, diverse disturbance scenarios are integrated simultaneously. The inlet temperature fluctuations are simulated with a sinusoidal oscillation of $+/-5 \mathrm{~K}$ around its nominal value of $298 \mathrm{~K}$ and a period duration of 1,500 seconds. In order to include the impact of the catalyst activity decay, an initial catalyst activity is considered with $a(t=0)=100 \%$ and a specific decay rate of $K_{\text {decay }}=0,000006$.

Thus, a gradual deactivation of the catalyst is simulated with a final reduced activity of $70 \%$ at the process end. Moreover, white noise with a maximum deviation of $8 \%$ for the component amounts and $2 \%$ for the temperature are assumed. The time constant of the filter is 15 seconds.

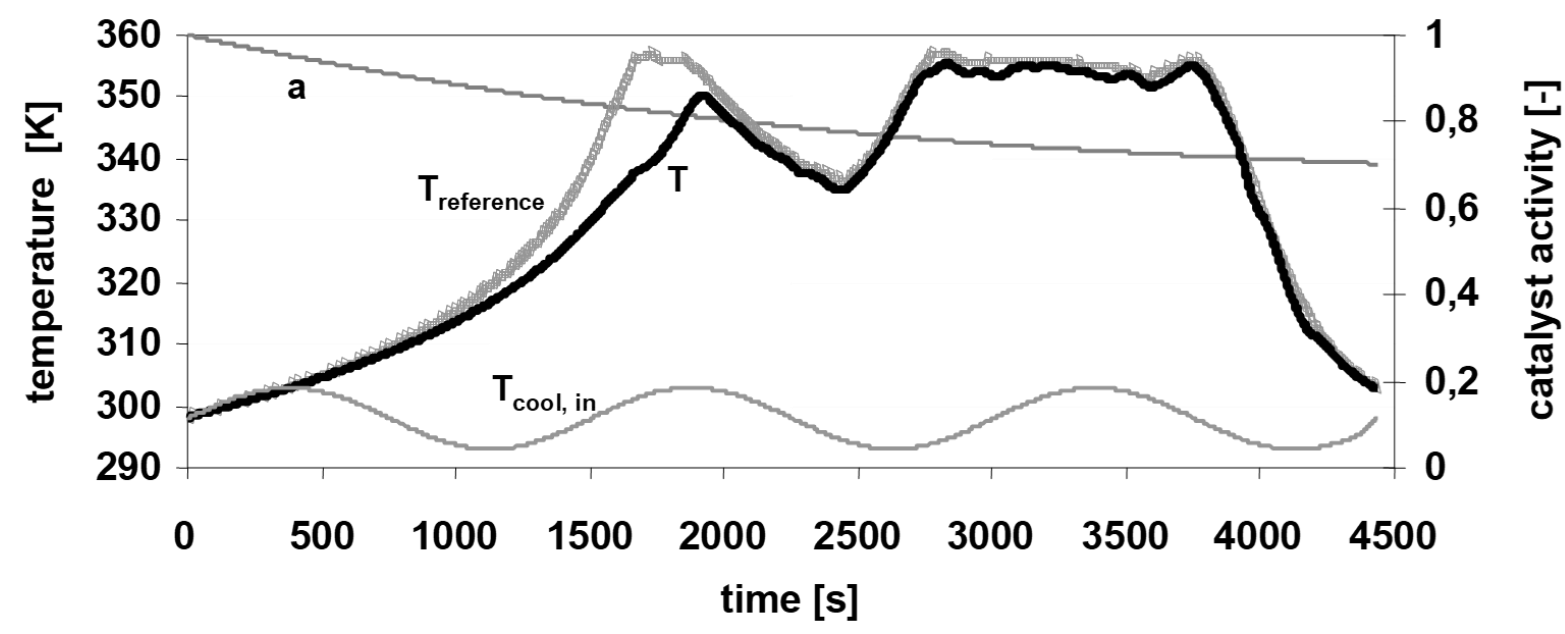

Figure 24: NMPC-based control of the exothermic fed-batch reactor under several disturbances without safety restrictions 


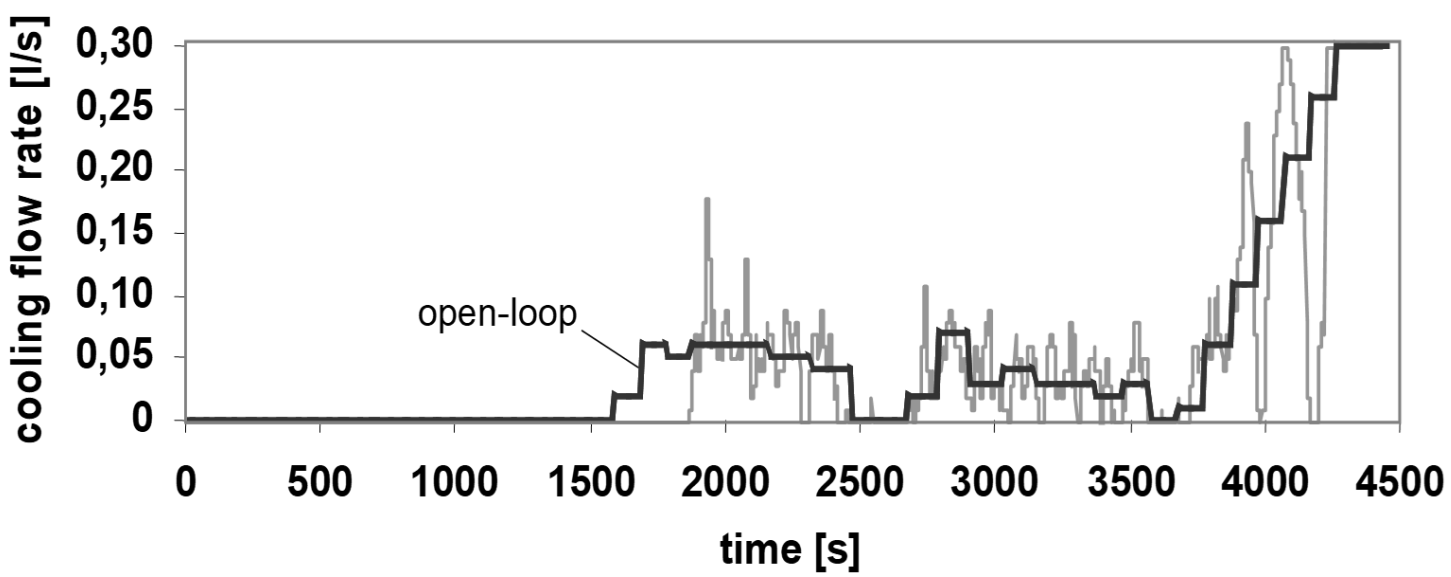

Figure 25: Trajectories of the cooling flow rate with regard to open and closed-loop optimisation for the first scenario

Despite several disturbances, the NMPC simulation results show almost perfect tracking regarding the reference temperature, in particular, after 2,000 seconds from the start of the process. The deviations at the beginning are due to the lower catalyst activity in combination with a smaller heat development as assumed in the open-loop optimisation. Thus, the difference between the reference trajectory and the actual reactor temperature is not based on the control activity. On the contrary, the controller responds rather appropriately extending the heating period until the reference trajectory is reached.

However, without considering safety restrictions the implemented controller features some deficiencies. Figures 26 and 27 show the results for another scenario using the same parameters from Table 2 . In this case study, the inlet cooling temperature $\left(T_{\text {cool, in }}=301 \mathrm{~K}\right)$ and the catalyst activity, $a=105 \%$ are assumed to be time-invariant representing the case of model mismatch.

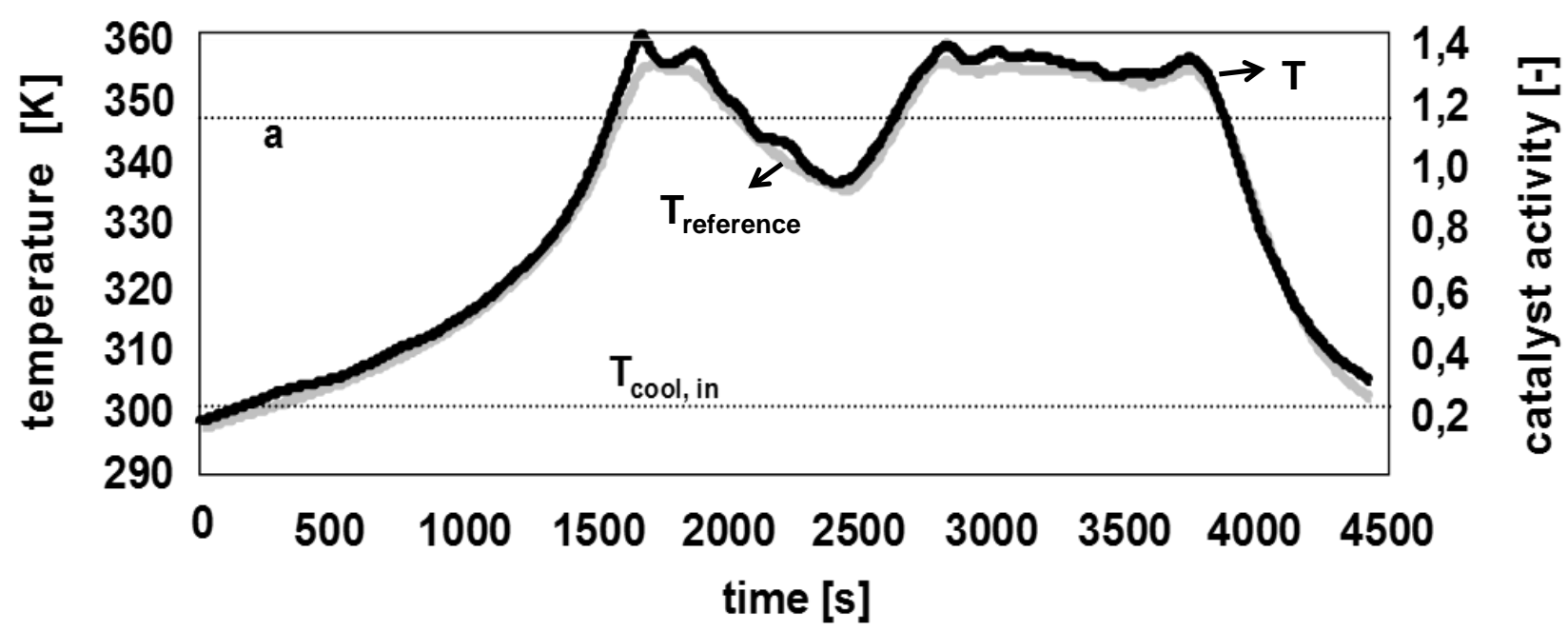

Figure 26: NMPC-based control with time-invariant values for the catalyst activity and the inlet cooling temperature 


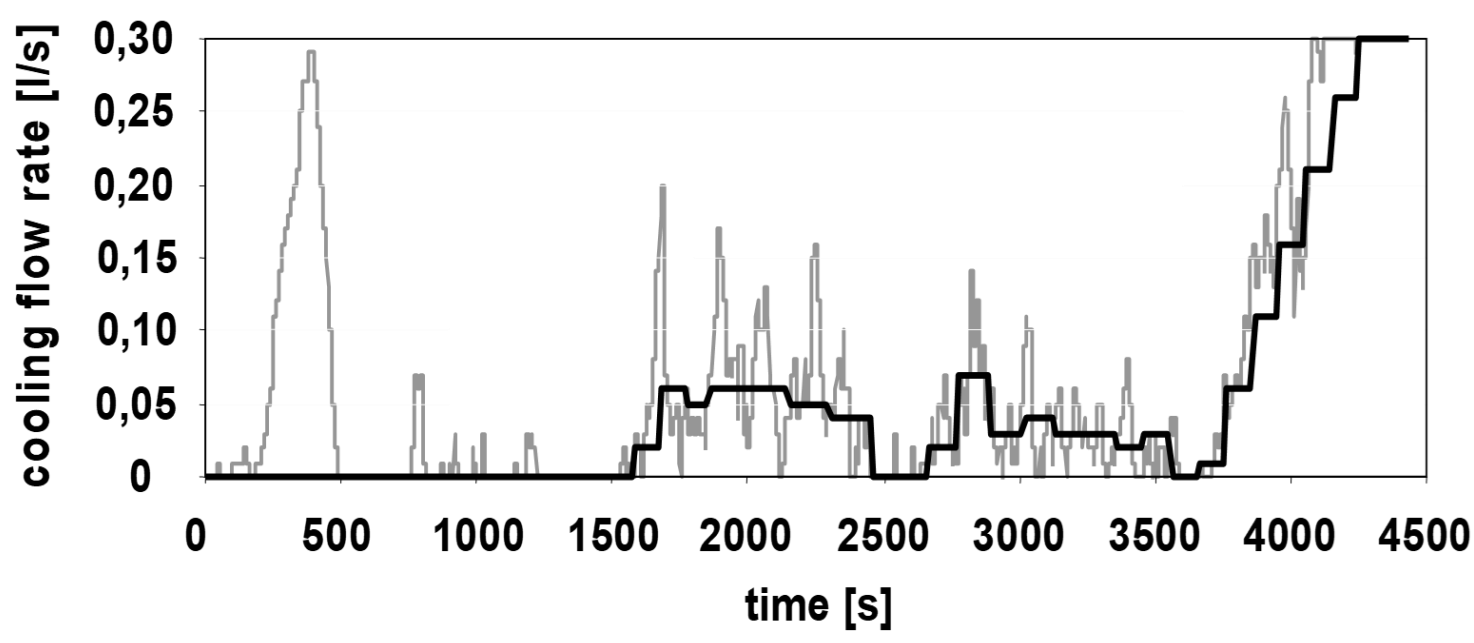

Figure 27: Trajectories of the cooling flow rate with regard to open and closed-loop optimisation for the second scenario

As a consequence, the reactor temperature is generally higher than the one in Figure 24. This is due to the controller model errors. The controller assumes a lower heat development and a higher cooling capacity than the ones actually arising in the implementation.

At the beginning of the heating process, the cooling pump is activated although the inlet cooling medium temperature still runs above the reactor temperature (Figure 27). In general, during the entire batch process the reactor temperature often lies above the reference trajectory and, as a result, it also exceeds the maximum allowed reactor temperature. This means that the reactor is operated in a forbidden region, where safety restrictions are not fulfilled.

Consequently, the controller quality essentially depends on the model accuracy. In other simulated scenarios even a runaway cannot be avoided. However, in a typical batch, process variables swing over wide ranges and process dynamics go through significant changes. Moreover, batch processes are characterised by significant uncertainties, a number of noisy measurements, and the fact that the controlled properties are typically not measured on-line. Therefore, the potential advantages one expects of a MPC system are likely to lead to be cancelled due to the impact of uncertainties, leading to significant tracking errors. Thus, in order to guarantee that the determined optimal operation remains safe, safety restrictions are needed to be incorporated explicitly in the control scheme.

\subsection{Dynamic adaptive back-off strategy simulation results}

In this section, the safety restrictions described in Section 4.1 are now explicitly considered in the NMPC-based control of the exothermic batch process including Equations (24) and (25).

On the one hand, an end constraint on the adiabatic temperature which guarantees that the temperature evolution does not exceed a critical safety limit, even in a case of cooling system failure. On the other hand, an upper limit on the reactor temperature which assures operability within specified operating regimes, according to the maximum available pump performance. Both 
constraints are to be enforced during the entire batch time and are formulated as hard-constraints in the optimisation problem.

In the nominal optimisation of the exothermic fed-batch reactor, safety restrictions have been considered. They are formulated both as path and end time point constraints. The open-loop resulting trajectories of the reactor temperature and the adiabatic end temperature are depicted in Figures 15 and 16. It can be observed that during a large part of the batch processing duration both states variables evolve along the upper limit. The adiabatic end temperature, in particular, is an active constraint over a large time period. Even though operation at this optimum is preferred, it usually cannot be accomplished with simultaneous fulfilment of all constraints, due to the effect of external disturbances. Thus, in this section, an NMPC based approach is proposed to implement such an optimal strategy remaining safe despite disturbances.

As previously illustrated in Figures 15 and 16, the true process optimum lies on the boundary of the feasible region defined by the active constraints. Thus, the safety constraints for the adiabatic end temperature and the reactor temperature within the moving horizon are now reformulated as follows:

$T(j) \leq 365-\tilde{T}_{\max } \cdot \alpha^{(j-2)}$

$T_{a d}(j) \leq 365-\widetilde{T}_{a d, \max } \cdot \alpha^{(j-2)}$

With $j=2, \ldots, 8 ; \alpha=0.5 ; \widetilde{T}_{\max }=4 \mathrm{~K} ;$ and $\tilde{T}_{a d, \max }=3 \mathrm{~K}$.

For the formulation of the NMPC-based online optimisation, the parameters of the objective function (28) are also taken from Table 2. The hard-constraints and their back-offs, which are now to be included in the optimisation problem are formulated in Equations (31) and (32), respectively. The manipulated variable is again the cooling flow rate. In order to compare the performances of the open-loop nominal solution and the nominal NMPC with the proposed dynamic adaptive backoff strategy, different disturbances have been considered.

In order to show the relevance of the developed closed-loop optimisation framework, the robustness of the developed strategies considering the safety restrictions is illustrated in Figures 28 and 29. The optimal policies guarantee the constraints compliance both for nominal operation as well as for cases of large disturbances (e.g., sudden cooling failure at any time-point). To emphasise this fact, diverse strong disturbances have been realised simultaneously. The inlet cooling temperature fluctuations are simulated with a sinusoidal oscillation of $+/-5 \mathrm{~K}$ around its 
nominal value of $298 \mathrm{~K}$ and a period duration of 1,500 seconds.

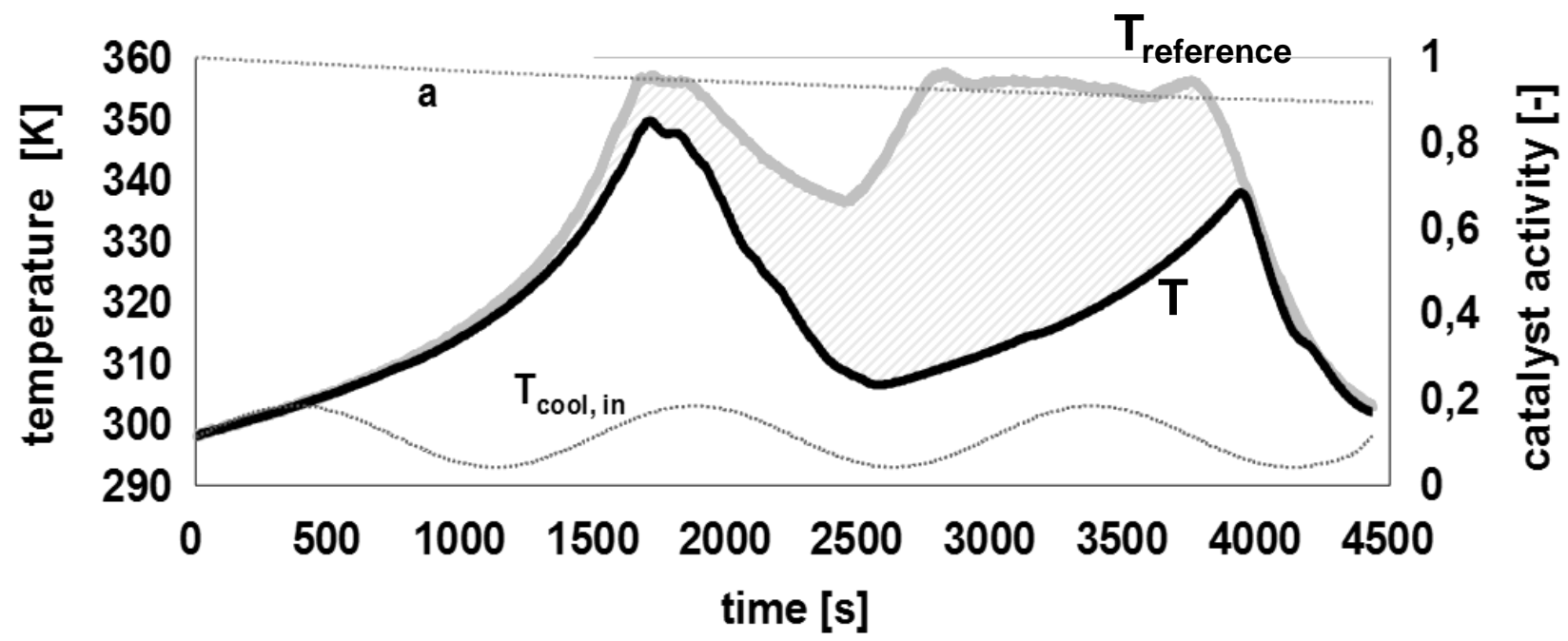

Figure 28: NMPC-based control of the exothermic fed-batch reactor considering safety restrictions under several disturbances

Moreover, an initial catalyst activity is considered with a $(t=0)=100 \%$ and a specific decay rate of $K_{\text {decay }}=0.0000015$. Thus, the final reduced activity is $86 \%$ at the process end. Moreover, all measurements are corrupted with white noise with a maximum deviation of $2 \%$ for the temperature and $8 \%$ for the component amounts.

As depicted in Figure 28, the reactor temperature evidently differs from the reference temperature (hashed area). Due mainly to the lower reaction performance, the reactor temperature fails to keep up with the reference temperature during the heating process.

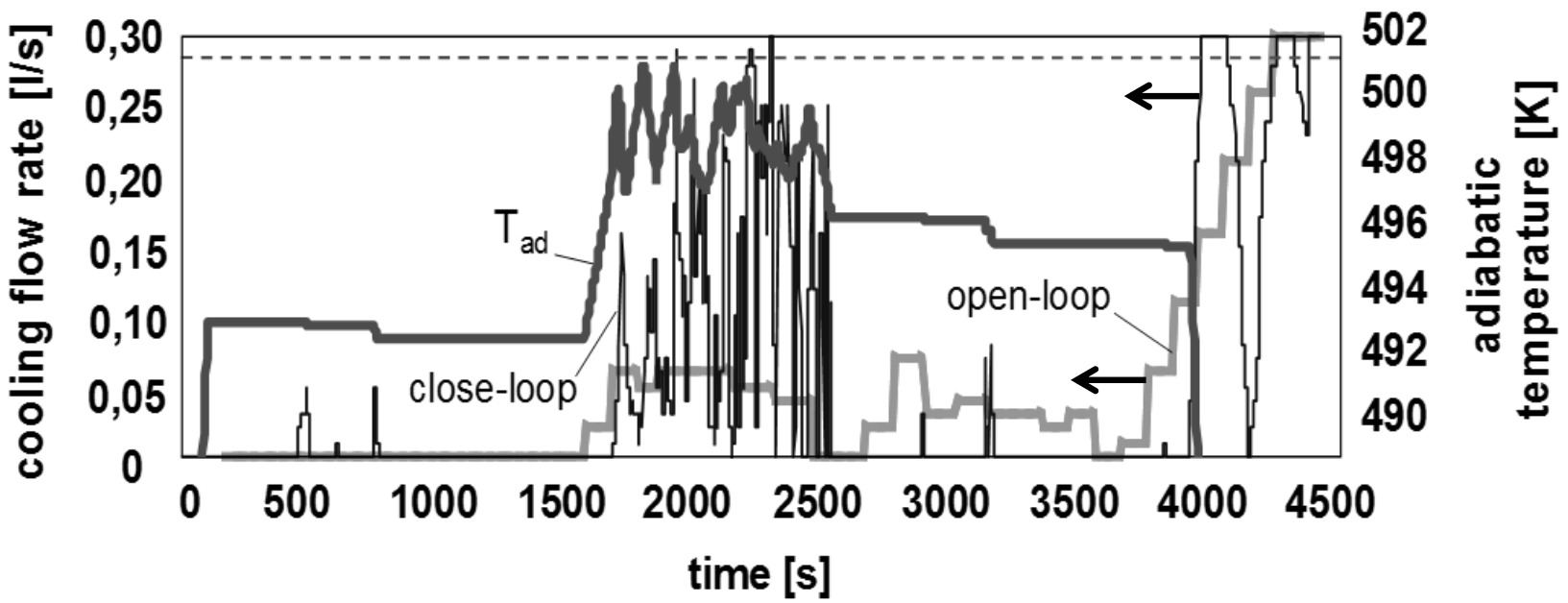

Figure 29: Optimal trajectory of the adiabatic temperature and the cooling flow rate with regards to open and closed-loop optimisation

Moreover, since the controller does not have a direct influence on the feed supply, in case of imminent danger of exceeding the adiabatic end temperature, the controller can only lower the reactor temperature. Thus, as shown in Figure 29, $T_{a d}$ reacts very sensitively during the time period when the feed flow is mainly supplied $(1,500-2,500 \mathrm{~s})$. Furthermore, since the heat 
removal and the trajectory of the feed flow rate are determined in such a way that for a large part of the operating time the process is run close to the feasible bounds, a higher activity of the controller can be observed in Figure 29. Thus, as the manipulated variable changes several peaks arise, which appear within those ranges where the constraints are active. These peaks are mostly caused by measurement noise. This is also the result of increasing the educt amount in the reactor and thus raising the potential in the reactor. Although in the reference trajectory a temperature reduction is provided, it is however not sufficient since a large amount of the educt has been accumulated due to the slower reaction as originally assumed. However, the constraint with regard to the adiabatic end temperature will then be violated if the reactor temperature is not lowered. The now lowered reactor temperature implies though a diminished reaction performance such that this effect will again be strengthened.

The explicit inclusion of the safety restrictions has both advantages and disadvantages. The main advantage is obviously the guarantee of compliance with the safety regulations at each time point during the operation. In general, the consideration of output constraints leads to an increased activity of the manipulated variable. This is due to the relatively short horizon of the controller and the relatively large influence of the uncertainties. However, the temperature control is effectively implemented and the process is now robust regarding the compensation for fast disturbances and, thus, guaranteeing operability within specified operating regimes. The highest priority is though given to the fulfilment of the safety restrictions. This implies that the operating conditions can quickly deviate from the reference conditions if there is any risk of exceeding the adiabatic end temperature. This arises primarily due to the changes in catalyst activity. In other words, the effects of slow disturbances or drifting parameters cannot be compensated satisfactorily. However, feasibility and robustness in particular with respect to output constraints have been

achieved by the presented dynamic backing-off strategy. On the other hand, in order to compensate for such disturbances, in the following Section the implementation of a second, higher level to the framework in Figure 6 is proposed (see Figure 7). This is definitely necessary since the selected operating conditions by the controller do not imply an optimal global solution for the operation. This is amongst others due to the rather limited control time horizon, the tracking of a given reference trajectory as well as the restricted actions which primarily concerns the cooling flow rate.

\subsection{A two-level strategy for optimisation based control}

In order to compensate slow disturbances, the on-line dynamic re-optimisation problem is automatically activated three times along the batch process time according to a trigger defined as the maximum allowable bounded difference, $\Delta T$, between the actual reactor temperature and the temperature reference trajectory (see Figure 30a).

New recipes resulting from this are then updated as input to the on-line framework. Due to the different trigger time-points the current D-RTO problem progressively possesses a reduced 
number of variables within a shrinking horizon (Nagy \& Braatz, 2003). However, as a result of this and a catalyst contamination, the final total batch time increases. But, despite the large model mismatch and the absence of kinetic knowledge, nearly perfect control is accomplished.

Thus, the resulting NMPC scheme embedded in the on-line re-optimisation framework is viable for the optimisation of the semi-batch reactor recipe while simultaneously guaranteeing the constraints compliance, both for nominal operation as well as for cases of large disturbances (e.g., cooling failure situation at any time-point). The proposed scheme yields almost the same profit as the one of the off-line optimisation operational profiles (see

Table 3).

Table 3: Nominal optimal and NMPC simulation results under the consideration of several uncertainties and disturbances

\begin{tabular}{lccc} 
& $C B_{f}[\mathrm{~mol}]$ & $C C_{f}[\mathrm{~mol}]$ & $t_{f}[\mathrm{~s}]$ \\
\hline Nominal open-loop optimisation & 152.5 & 37.8 & 4,434 \\
\hline NMPC / unconstrained & 141.0 & 28.8 & 4,434 \\
\hline NMPC / safety restrictions / dynamic back-off strategy & 127.9 & 12.8 & 4,434 \\
\hline NMPC / safety restrictions / dynamic back-off / D-RTO & 148.8 & 36.8 & 4,892
\end{tabular}

The real output variables and the current estimated catalyst activity are handed over to the reoptimisation step as constant values for the triggered optimisation run the corresponding timepoint. The information about the remaining or already employed educt $A$ is crucial. This issue changes the total amount of $\mathrm{A}, n_{A, t o t a l}$, to be still supplied and, therefore, the equality constraint, defined in Equation (22), needs to be readjusted.

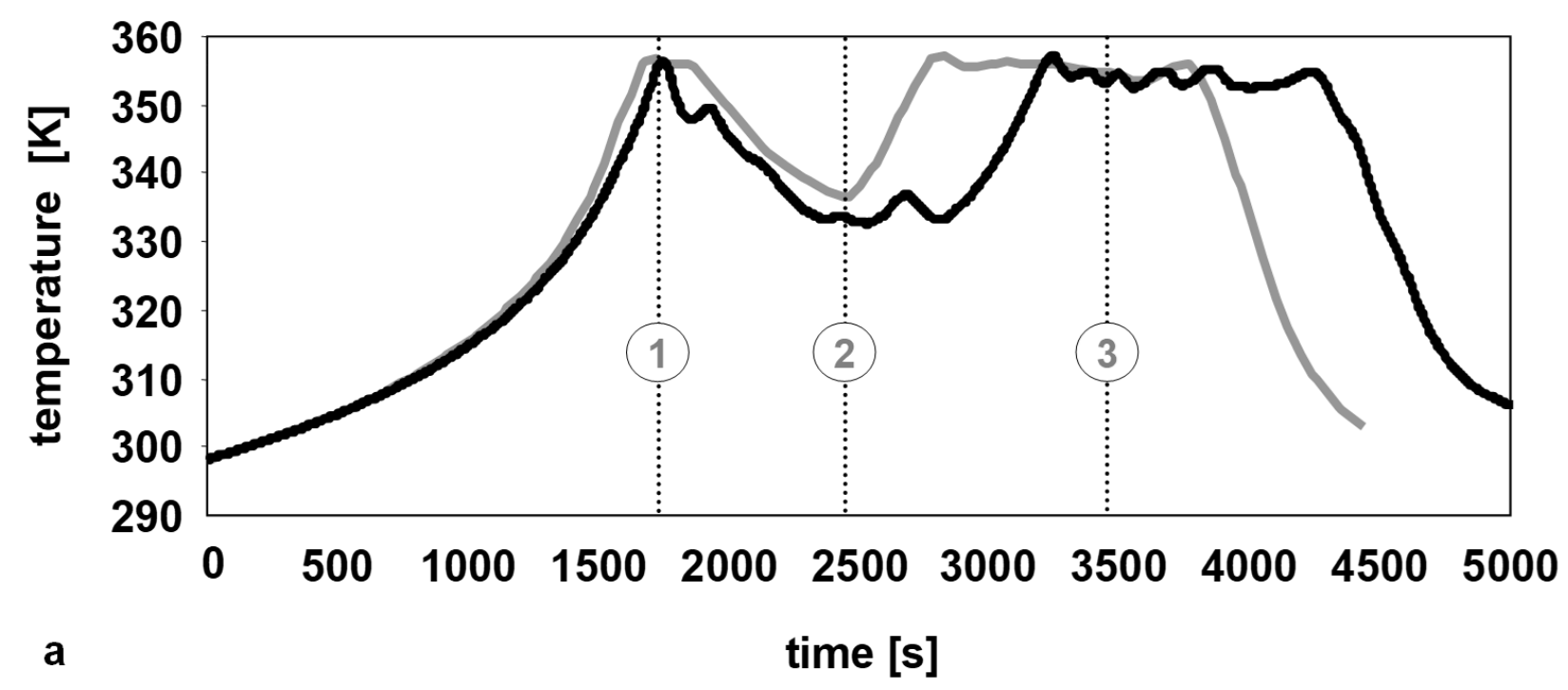



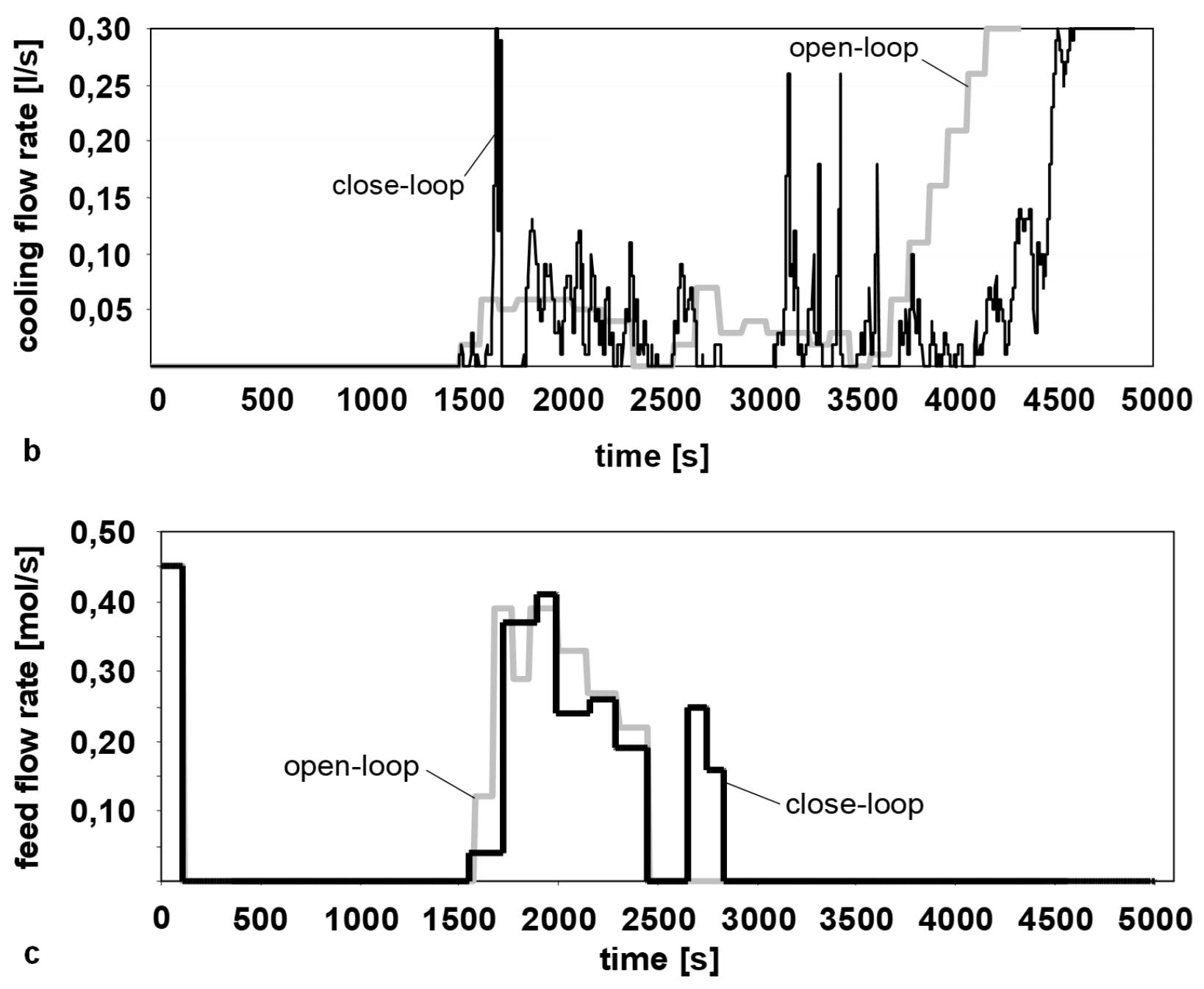

Figure 30: Implementation results of the online re-optimisation: a) Reference and optimal reactor temperature; b) Open and closed-loop optimal cooling flow rate; c) Open and closedloop optimal feed flow rate

However, both the online optimisation and the controller use the imperfect model as also assumed in the open-loop optimisation (Section 2.5.1). In Figure 30 (a-c), the implementation results of the online optimisation in comparison with the nominal open-loop optimisation are shown.

The resulting new reference trajectories are updated three times during the batch run, at 1,700, 2,450 and 3,450 seconds. The different strong disturbances considered are again the oscillation of the inlet cooling temperature, a catalyst contamination with $K_{\text {decay }}=0,0000021$, as well as perturbed measurements with white noise. The objective function of the D-RTO is the same as the one defined in Equation (21). From the results in Figure 30 (a-c) it is evident that a substantial improvement of the product yield can be obtained by re-optimising the operating conditions. However, the required total time of operation has increased. An alternative to counter this problem might be to adapt suitably or restate the objective function of the D-RTO based on the new arising process conditions.

\subsection{Robust chance-constrained NMPC under uncertainty}


The performance objective function from Equation (33) is now redefined as follows:

$\min _{V_{\text {cool }}}\left(N_{U}\right)=\sum_{k=N_{1}}^{N_{U}}[\Delta u(t+k-1)]^{2}$

This objective is subject to the entire first principles model as described in Section 2.1. In addition, in order to compare the performance of the chance constrained NMPC with the dynamic adaptive back-off strategy, the constraint regarding the process shut down is not included in the nominal open-loop optimisation. Furthermore, while the hard-constraint with regard to the adiabatic end temperature in Equation (32) is still included, the safety restriction in Equation (31) corresponding to the maximum allowable reactor temperature is now formulated as a chance constraint within the moving horizon:

$\operatorname{Pr}\left\{T(k+i \mid k) \leq T_{\max }=365 K\right\} \geq \alpha$

The decision variable here is the cooling flow rate as well.By solving the problem in Equation (34), a value of $\alpha^{\max }=96.7 \%$ is achieved for the runaway reaction case study considered in this work. In order to identify the potential of the proposed approach, the main disturbance assumed is the catalyst activity with a variance of $15 \%$.

However, the use of this strategy for a transient process with the consideration of uncertainties in advance has a great impact for those NMPC problems where the reference trajectory is very close to a defined upper bound of the constrained output at some time-periods. In order to find further improvement of the operation due to the stochastic approach, the chance-constrained approach and the deterministic dynamic adaptive back-off strategy are compared.

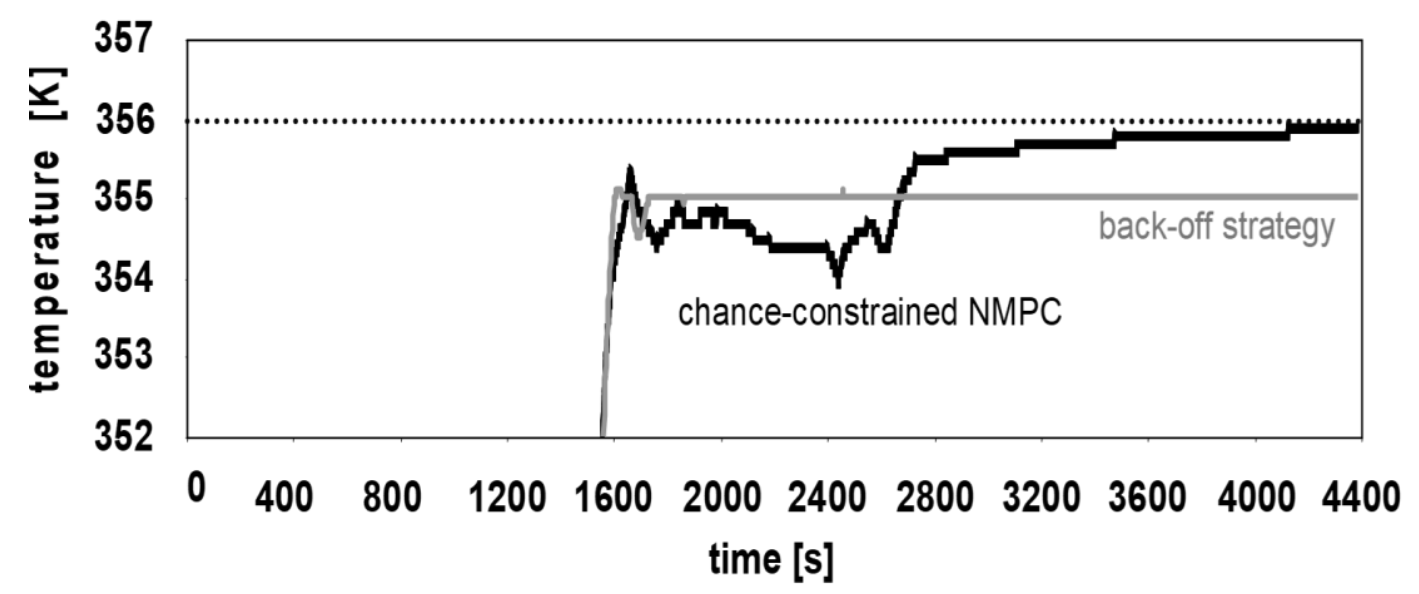

Figure 31: Reactor temperature trajectory for the back-off and chance-constrained NMPC strategies

The resulting trajectories of the reactor temperature concerning both strategies are illustrated in Figure 31. It can be seen that the reactor temperature trajectory based on the back-off strategy reaches early a stationary value caused by the fixed bounds of the temperature formulated in the corresponding optimisation problem. The temperature curve of the chance-constrained approach shows several changes with lower values of temperatures compared to the back-off strategy just 
after the heating period, and higher values after the total feed amount has been supplied (see Figure 32). This is caused by the fact that, when accounting for uncertainties in advance, the stochastic approach takes into consideration the reactor's temperature sensitivity to uncertain parameters.

Due to the higher sensitivities, the stochastic approach implements a more conservative strategy after 1,600 seconds, and, thus, the operation may achieve more robustness than the back-off strategy. Towards the end of the process, the decrease of sensitivities is used for a close approach to the maximum allowable reactor temperature and thus leading to a better objective value.

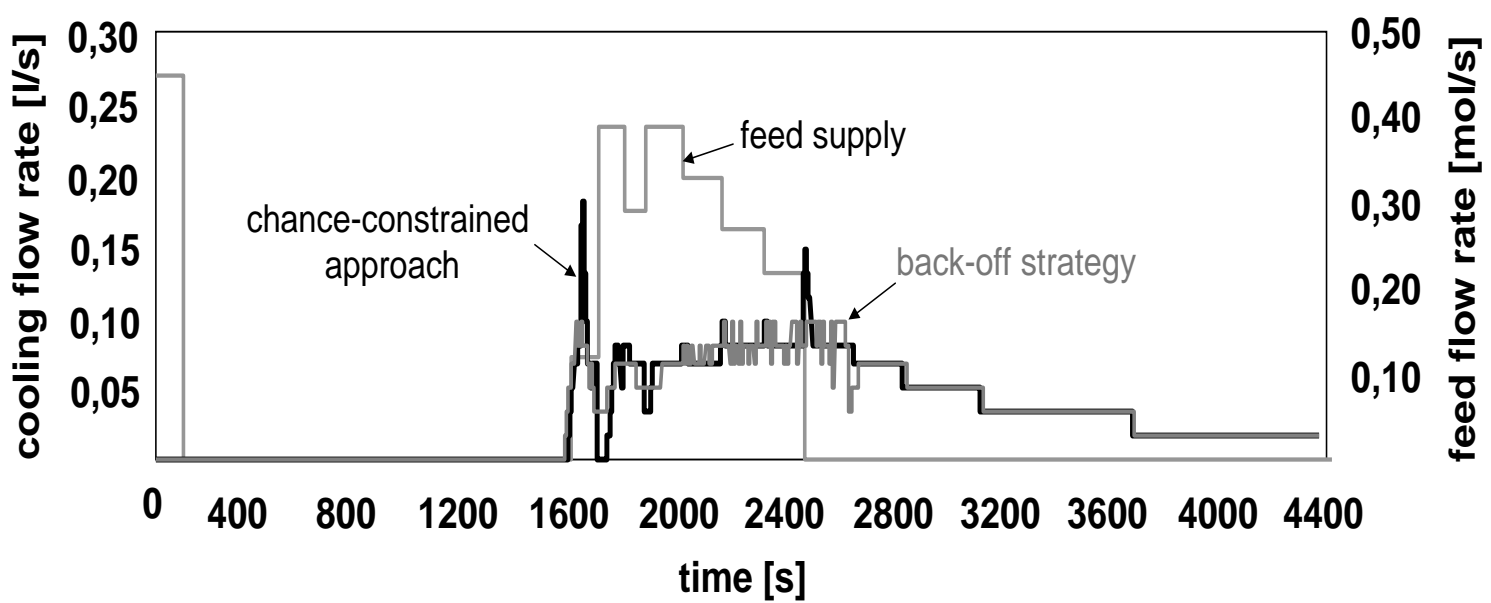

Figure 32: Optimal cooling flow rate for the back-off and chance-constrained strategies and the given feed supply

Moreover, different confidence levels can be assigned to different time periods within the moving horizon by using single chance constraints. Consequently, a decreasing factor (i.e., a lower confidence level, $\alpha$ for the future periods within the horizon) can be introduced. As a result, the process operation will be as close as possible to the constrained boundaries. Thus, the chanceconstrained strategy leads to an improvement of both robustness and the objective value. Furthermore, it enables a better quantification of how to implement a tuneable policy of satisfaction of the underlying safety constraints (how to get close to them). The chanceconstrained strategy would allow for a better optimisation objective function value due to the constraints being less conservatively over-satisfied, as is the case of the back-off heuristic policy.

\section{Conclusions}

Model-based process control of transient processes has become very important for industrial applications during the last few decades. However, transient processes are inherently dynamic and are characterised by the fact that some of the controlled properties are commonly not measured on-line. Moreover, since nonlinear models are derived from input-output data, which inevitably contain significant bias and variance, the uncertainties and disturbances are required to be quantified and considered explicitly in the controller design and analysis. As demonstrated 
for the semi-batch reactor under safety restrictions, the potential advantages of a model-based control system are otherwise likely to lead to significant tracking errors if these uncertainties and disturbances are not rigorously quantified and included in the optimisation problems involved for controlling the underlying processes.

In this work, two methods are introduced which are based on nonlinear model predictive control (NMPC) schemes to solve closed-loop dynamic optimisation problems in an online framework. The key idea lies in the consideration of unknown and unexpected disturbances in advance (i.e. anticipating, in particular, violation of output hard-constraints, which are strongly affected by instantaneous disturbances).

The first method introduced is realised by means of an adaptive backing off of their bounds along the moving horizon with a decreasing degree of severity with increasing time, leading then to the generation of a trajectory consisting of the modified constraint bounds. However, this trajectory is dependent on the amount of measurement error and parameter variation, including uncertainty. In addition, for the integration of dynamic real-time optimisation and control of transient processes, a two-stage strategy is considered which is characterised by a second, higher level corresponding to a dynamic optimisation problem and a first, lower level related to a tracking control problem.

The second method considers the chance-constrained control approach, where the known properties of some major disturbances can be integrated in the NMPC formulation. These are described with stochastic distributions, which can be estimated from historical data. Moreover, the influence of the uncertain variables on the output constraints propagates through the nonlinear process with the evolution in time. The solution of the chance-constrained NMPC problem has the features of prediction, robustness and being closed-loop. Due to the properties of the moving horizon, the developed control strategy is extended to on-line optimisation under uncertainty. Thus, a novel concept based on a nonlinear MPC scheme has been introduced to solve closedloop stochastic dynamic optimisation problems assuring efficiently both robustness and feasibility with respect to input and, in particular, to output constraints. The formulation of individual predefined probability limits of complying with the restrictions incorporates the issue of feasibility and the contemplation of a trade-off between profitability and reliability.

In order to demonstrate the performance of the developed concepts and the efficiency of the proposed online framework, both schemes are applied for the on-line optimisation of a semi-batch non-isothermal reactor under safety (hard-) constraints and the influence of several disturbances.

In terms of future work, the back-off scheme is proposed to be developed further in terms of a theoretical-algorithmic point of view, as a first step. In particular, there is no rigorous scheme as yet by which the back-off envelopes of bounds on process outputs can be tailored so as to have a predefined impact on the process performance. Nor have there been any special methodologies been proposed in the open literature to design these envelopes of tighter bounds so as to guarantee a level of satisfaction of constraints in the presence of uncertainties and 
disturbances with known statistical properties. Thus this is a first important aspect we wish to explore in the near future, so as to provide rigorous theoretical analysis for its properties, and practical implementation guidelines which will be very important for practical industrial applicability.

The second area we would like to explore, in particular within the framework of implementing chance-constrained schemes, as per our second proposed method in this work, is intensification of batch systems with the inclusion of newly presented theoretical work on MPC schemes with stability constraints, such as in the recently published original work by Kähm \& Vassiliadis (2018a, 2018b, 2018c).

In conclusion, we are confident that the work presented in this paper constitutes not only a novel and integrated approach towards dealing with online advanced control algorithms, but also to extend them to include process uncertainties in a rigorous and satisfactory manner, paving the way for a multitude of novel contributions soon.

\section{References}

Abel, O., Helbig, A., Marquardy, W., Zwick, H., Daszkowski, T., 2000, Productivity optimisation of an industrial semi-batch polymerisation reactor under safety constraints, Journal of Process Control 10 (4), 351-362

Alessio, A. \& Bemporad, A., 2009, A survey on explicit model predictive control, in Nonlinear model predictive control. Lecture notes in control and information sciences, vol. 384, Springer, Berlin, 345-369

Allgöwer, F., Badgwell, T.A., Qin, J.S., Rawlings, J.B., Wright, S.J., 1999, Nonlinear predictive control and moving horizon estimation - An introductory overview, in Advances in Control, Springer, London, 391-449

Arellano-Garcia, H. \& Wozny, G., 2009, Chance constrained optimisation of process systems under uncertainty: I. Strict monotonicity, Computers and Chemical Engineering 33 (10), 15681583

Aydin, E., Bonvin, D., Sundmacher, K., 2018, NMPC using Pontryagin's minimum principleapplication to a two-phase semi-batch hydroformylaton reactor under uncertainty, Computers and Chemical Engineering 108, 47-56

Barz, T., Wozny, G., Arellano-Garcia, H., 2011, Robust implementation of optimal decisions using a two-layer chance-constrained approach, I\&EC Research 50 (9), 5050-5063

Bemporad, A. \& Morari, M., 1999, Robust model predictive control: A survey, in Robustness in identification and control, Springer, London, 207-226

Bonvin, D. \& François, 2017, Control and optimisation of batch chemical processes, in Coulson and Richardson's Chemical Engineering, Fourth Edition, Vol. 3b: Process Control, 441-503 
Camacho, E.F. \& Bordons, C., 1999, Model predictive control, Springer, Berlin

Chischi, L., Rossiter, J.A., Zappa, C., 2001, Systems with persistent disturbances: predictive control with restricted constraints, Automatica 37 (7), 1019-1028

Darby, M.L. \& Nikolaou, M., 2012, MPC: Current practice and challenges, Control Engineering Practice 20, 328-342

De Nicolao, G., Magni, L., Scattolini, R., 2000, Stability and robustness of nonlinear receding horizon control, in Nonlinear Model Predictive Control. Progress in systems and control theory, Vol. 26, Birkhäuser, Basel, 3-22

Dubljevic, S., Mhaskar, P., El-Farra, N.H., Christofides, P.D., 2005, Predictive control of transportreaction processes, Computers and Chemical Engineering 29, 2335-2345

Ellis, M., Durand, H., Christofides, P.D., 2014, A tutorial review of economic model predictive control methods, Journal of Process Control 24, 1156-1178

Emenike, V.N., Xie, X., Schenkendorf, R., Spiess, A.C., Krewer, U., 2019, Robust dynamic optimisation of enzyme-catalyzed carboligation: A point estimate-based back-off approach, Computers and Chemical Engineering 121, 232-247

Fogler, H.S., 1999, Elements of chemical reaction engineering, Prentice Hall Int. London

Forbes, M.G., Patwardhan, R.S., Hamadah, H., Gopaluni, R.B., 2015, Model predictive control in industry: Challenges and opportunities, IFAC-PapersOnLine 48-8, 531-538

Galvanin, F., Barolo, M., Bezzo, F., Macchietto, S., 2009, A backoff strategy for model-based experiment design under parametric uncertainty, AIChE Journal 56 (8), 2088-2102

Grifith, D.W., Biegler, L.T., Pathwardhan, S.C., 2018, Robustly stable adaptive horizon nonlinear model predictive control, Journal of Process Control 70, 109-122

Haseltine, E.L. \& Rawlings, J.B., 2005, Critical evaluation of extended Kalman filtering and moving-horizon estimation, I\&EC Research 44 (8), 2451-2460

Helbig, A., Abel, O., Marquardt, W., 1998, Model predictive control for on-line optimisation of semibatch reactors, Proceedings of the 1998 American Control Conference, 1695-1699

Kähm, W. \& Vassiliadis, V. S., 2018, Optimal Lyapunov Exponent Parameters for Stability Analysis of Batch Reactors with Model Predictive Control, Computers and Chemical Engineering 119, 270-292 (2018a)

Kähm, W. \& Vassiliadis, V. S., 2018, Stability criterion for the intensification of batch processes with model predictive control, Chemical Engineering Research and Design 138, 292-313 (2018b) Kähm, W. \& Vassiliadis, V. S., 2018, Thermal stability criterion integrated in model predictive control for batch reactors", Chemical Engineering Science 188, 192-207 (2018c) 
Koller, R.W., Ricardez-Sandoval, L.A., Biegler, L.T., 2018, Stochastic back-off algorithm for simultaneous design, control and scheduling of multiproduct systems under uncertainty, AIChE Journal 64(7), 2379-2389

Kothare, M.V., Balakrishnan, V., Morari, M., 1996, Robust constrained model predictive control using linear matrix inequalities, Automatica 32 (10), 1361-1379

Lee, J.H., 2011, Model predictive control: Review of the three decades of development, International Journal of Control, Automation and Systems 9 (3), 415-424

Lee, J.H. \& Yu, Z., 1997, Worst-case formulations of model predictive control for systems with bounded parameters, Automatica 33 (5), 763-781

Li, P., Arellano-Garcia, H., Wozny, G., Reuter, E., 1998, Optimisation of a semibatch distillation process with model validation on the industrial site, Industrial and Engineering Chemistry Research 37, 1341-1350

Li, P., Wendt, M., Wozny, G., 2002, A probabilistically constrained model predictive controller, Automatica 38 (7), 1171-1176

Li, P., Arellano-Garcia, H., Wozny, G., 2008, Chance constrained programming approach to process optimisation under uncertainty, Computers and Chemical Engineering 32 (1-2), 25-45

Loeblein, C. \& Perkins, J.D., 1999, Analysis and structural design of integrated on-line optimisation and regulatory control systems, AIChE Journal 45 (5), 1030-1040

Logist, F., Houska, B., Diehl, M., Van Impe, J.F., 2011, Robust multi-objective optimal control of uncertain (bio)chemical processes, Chemical Engineering Science 66, 4670-4682

Ma, D.L., Chung, S.H., Braatz, R.D., 1999, Worst-case performance analysis of optimal batch control trajectories, AIChE Journal 45 (7), 1469-1476

Maussner, J., Freund, H., 2018, Efficient calculation of constraint back-offs for optimisation under uncertainty: A case study on maleic anhydride synthesis, Chemical Engineering Science 192, 306-317

Mayne, D.Q., 2000, Nonlinear model predictive control: Challenges and opportunities, in Nonlinear model predictive control. Progress in systems and control theory, Vol. 26, Birkhäuser, Basel, 23-44

Mayne, D.Q., Rawlings, J.B., Rao, C.V., Scokaert, P.O.M., 2000, Constrained model predictive control: Stability and optimality, Automatica 36, 789-814

Mayne, D.Q., 2014, Model predictive control: Recent developments and future promise, Automatica 50, 2967-2986

Mayne, D.Q., 2016, Robust and stochastic model predictive control: Are we going in the right direction?, Annual Reviews in Control 41, 184-192 
Mhaskar, P., El-Farra, N.H., Christofides, P.D., 2005, Robust hybrid predictive control of nonlinear systems, Automatica 41 (2), 209-217

Morari, M. \& Lee, J.H., 1999, Model predictive control: past, present and future, Computers and Chemical Engineering 23, 667-682

Nagy, Z.K. \& Braatz, R.D., 2003, Robust nonlinear model predictive control of batch processes, AIChE Journal 49 (7), 1776-1786

Nagy, Z.K. \& Braatz, R.D., 2004, Open-loop and closed-loop robust optimal control of batch processes using distributional and worst-case analysis, Journal of Process Control 14, 411-422

Pahija, E., Manenti, F., Mujtaba, I.M., 2013, Optimisation of batch and semi-batch reactors, Computer Aided Chemical Engineering 32, 739-744

Pushke, J., Zubov, A., Kosek, J., Mitsos, A., 2017, Multi-model approach based on parametric sensitivities - A heuristic approximation for dynamic optimisation of semi-batch processes with parametric uncertainties, Computers and Chemical Engineering 98, 161-179

Qin, S.J., Badgwell, T.A., 2003, A survey of industrial model predictive control technology, Control Engineering Practice 11, 733-764

Saltik, M.B., Özkan, L., Ludlage, J.H.A., Weiland, S., Van den Hof, P.M.J., 2018, An outlook on robust model predictive control algorithms: Reflections on performance and computational aspects, Journal of Process Control 61, 77-102

Schwarm, A.T. \& Nikolaou, M., 1999, Chance-constrained model predictive control, AIChE Journal 45 (8), 1743-1752

Shi, J., Biegler, L.T., Hamdan, I., Wassick, J., 2016, Optimisation of grade transitions in polyethylene solution polymerisation process under uncertainty, Computers and Chemical Engineering 95, 260-279

Soliman, M., Swartz, C.L.E., Baker, R., 2008, A mixed-integer formulation for back-off under constrained predictive control, Computers and Chemical Engineering 32, 2409-2419

Visser, E., Srinivasan, B., Palanki, S., Bonvin, D., 2000, A feedback-based implementation scheme for batch process optimisation, Journal of Process Control 10, 399-410

Wendt, M., 2005, Untersuchung zur stochastischen Online Optimierung kontinuierlicher Destillationsprozesse unter Unsicherheiten, PhD Thesis, Berlin University of Technology, Germany

Yu, S., Reble, M., Chen, H., Allgöwer, F., 2014, Inherent robustness properties of quasi-infinite horizon nonlinear model predictive control, Atuomatica 50, 2269-2280

Zavala, V.M. \& Biegler, L.T., 2009, The advanced-step NMPC controller: Optimality, stability and robustness, Automatica 45 (1), 86-93 